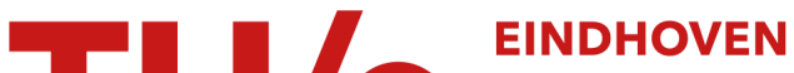 UNIVERSITY OF TECHNOLOGY
}

\section{Positivity properties of phase-plane distribution functions}

Citation for published version (APA):

Janssen, A. J. E. M. (1984). Positivity properties of phase-plane distribution functions. Journal of Mathematical Physics, 25(7), 2240-2252. https://doi.org/10.1063/1.526417

DOI:

10.1063/1.526417

Document status and date:

Published: 01/01/1984

\section{Document Version:}

Publisher's PDF, also known as Version of Record (includes final page, issue and volume numbers)

\section{Please check the document version of this publication:}

-A submitted manuscript is the version of the article upon submission and before peer-review. There can be important differences between the submitted version and the official published version of record. People interested in the research are advised to contact the author for the final version of the publication, or visit the $\mathrm{DOI}$ to the publisher's website.

- The final author version and the galley proof are versions of the publication after peer review.

- The final published version features the final layout of the paper including the volume, issue and page numbers.

Link to publication

\section{General rights}

Copyright and moral rights for the publications made accessible in the public portal are retained by the authors and/or other copyright owners and it is a condition of accessing publications that users recognise and abide by the legal requirements associated with these rights.

- Users may download and print one copy of any publication from the public portal for the purpose of private study or research.

- You may not further distribute the material or use it for any profit-making activity or commercial gain

- You may freely distribute the URL identifying the publication in the public portal.

If the publication is distributed under the terms of Article $25 \mathrm{fa}$ of the Dutch Copyright Act, indicated by the "Taverne" license above, please follow below link for the End User Agreement:

www.tue.nl/taverne

Take down policy

If you believe that this document breaches copyright please contact us at:

openaccess@tue.nl

providing details and we will investigate your claim. 


\title{
Positivity properties of phase-plane distribution functions
}

\author{
A. J. E. M. Janssen \\ Philips Research Laboratories, $5600 \mathrm{JA}$ Eindhoven, The Netherlands
}

(Received 1 November 1983; accepted for publication 16 February 1984)

\begin{abstract}
The aim of this paper is to compare the members of Cohen's class of phase-plane distributions with respect to positivity properties. It is known that certain averages (which are in a sense compatible with Heisenberg's uncertainty principle) of the Wigner distribution over the phaseplane yield non-negative values for all states. It is shown in this paper that the Wigner distribution is unique in this respect among the members of Cohen's class that have correct marginals or that satisfy Moyal's formula for all states. The subset of members of Cohen's class (not necessarily satisfying one of these two conditions) with positivity properties comparable with those for the Wigner distribution is shown to be rather small.
\end{abstract}

PACS numbers: 03.65. $-\mathrm{w}, 02.30 .+\mathrm{g}$

\section{INTRODUCTION}

In this Introduction we present in a rather informal way some known facts about Cohen's class of phase-plane distribution functions, and we indicate what we are aiming at in this paper. Cohen's class is parametrized by means of a function $\Phi$ of two variables : for any such $\Phi$ we have the family of phase-plane distribution functions

$$
\begin{aligned}
C_{f}^{(\Phi)}(q, p)= & \iiint \exp [-2 \pi i(\theta q+\tau p-\theta u)] \Phi(\theta, \tau) \\
& \times f\left(u+\frac{1}{2} \tau\right) \overline{f\left(u-\frac{1}{2} \tau\right)} d \theta d \tau d u \quad\left[(q, p) \in \mathbb{R}^{2}\right],
\end{aligned}
$$

where $f$ is an arbitrary state (all integrations are over the real line, unless indicated otherwise). Of course, in order for this definition to make sense certain assumptions on $\Phi$ as well as on $f$ should be made. In Sec. II a convenient mathematical setting for dealing with rather general $\Phi$ 's in (1.1) is presented. Any family $C_{f}^{(\Phi)}(f$ arbitrary state) can be used to give a formulation of quantum mechanics in the phase plane of position $q$ and momentum $p$. In fact, it can be shown that any bilinear map $f \rightarrow C_{f}$, mapping states $f$ onto functions $C_{f}$ of the phase-plane variables $(q, p)$, satisfying

$$
C_{f}(q+a, p+b)=C_{T_{a} R_{f}}(q, p) \quad\left[(q, p) \in \mathbb{R}^{2}\right]
$$

for all states $f$ and all $(a, b) \in \mathbb{R}^{2}$ can be brought into the form (1.1). Here $T_{a}$ and $R_{b}$ are the shift operators, defined, respectively, by

$$
\begin{aligned}
& \left(T_{a} f\right)(q)=f(q+a), \\
& \left(R_{b} f\right)(q)=e^{-2 \pi i b q} f(q) \quad(q \in \mathbb{R}),
\end{aligned}
$$

for all $f$ and all $(a, b) \in \mathbb{R}^{2}$. It is easily verified that any $C_{f}=C_{f}^{(\Phi)}$ as in (1.1) satisfies (1.2) for all $f$ and all $(a, b) \in \mathbb{R}^{2}$.

The choice $\Phi(\theta, \tau)=1$ in (1.1) yields the Wigner distribution $^{2}$ of $f$, viz.

$$
\begin{aligned}
& W_{f}(q, p)=\int e^{-2 \pi i p t} f\left(q+\frac{1}{2} t\right) \overline{f\left(q-\frac{1}{2} t\right)} d t \\
& {\left[(q, p) \in \mathbb{R}^{2}\right] .}
\end{aligned}
$$

In a way one can consider the Wigner distribution as the basic distribution of Cohen's class from which all others can be derived ${ }^{3}$ : one has

$$
\begin{gathered}
C_{f}^{(\Phi)}(q, p)=\iint \varphi(q-a, p-b) W_{f}(a, b) d a d b \\
{\left[(q, p) \in \mathbb{R}^{2}\right],}
\end{gathered}
$$

where $\varphi$ is the double Fourier transform of $\Phi$, given by

$$
\begin{gathered}
\varphi(q, p)=\iint e^{-2 \pi i \mid \theta q+\tau p)} \Phi(\theta, \tau) d \theta d \tau \\
{\left[(q, p) \in \mathbb{R}^{2}\right] .}
\end{gathered}
$$

This $\varphi$ must be treated as a generalized function, e.g., $\varphi(q, p)=\delta(q) \delta(p)$ for the Wigner distribution case, whereas $\Phi$ is usually smooth.

The class of all possible phase-plane distributions can be restricted considerably by imposing certain "natural" requirements. We consider in this paper four additional conditions.

(a) $C_{f}^{(\Phi)}$ yields the "correct" marginal distributions for all states $f$ [see (1.7)]. (1.12)].

(b) $C_{f}^{(\Phi)}$ has finite support properties [see (1.11) and

(c) $C_{f}^{(\Phi)}$ is such that Moyal's formula holds for all states $f$ and $g$ [see (1.15)].

(d) $C_{f}^{(\phi)}$ is a non-negative distribution for all states $f$. Each of the requirements (a), (b), (c), and (d) has consequences for $\Phi$ (and $\varphi$ ); it is well known that not all four conditions are compatible. However, the Wigner distribution satisfies (a), (b), and (c), while also certain positivity properties hold.

The condition (a) means that for all states $f$ we should have

$$
\begin{aligned}
& \int C_{f}^{(\Phi)}(q, p) d p=|f(q)|^{2} \quad(q \in \mathbb{R}), \\
& \int C_{f}^{(\Phi)}(q, p) d q=|(\mathscr{F} f)(p)|^{2} \quad(p \in \mathbb{R}) .
\end{aligned}
$$

Here $\mathscr{F}$ denotes the Fourier transform, given for all $f$ by

$$
(\mathscr{F} f)(p)=\int e^{-2 \pi i q p} f(q) d q \quad(p \in \mathbb{R}) .
$$

It can be shown ${ }^{1,3,4}$ that (1.7) holds for all states $f$ if and only if 


$$
\Phi(0, \tau)=\Phi(\theta, 0)=1 \quad\left[(\theta, \tau) \in \mathbb{R}^{2}\right],
$$

or, equivalently,

$$
\begin{aligned}
& \int \varphi(q, p) d p=\delta(q) \quad(q \in \mathbb{R}), \\
& \int \varphi(q, p) d q=\delta(p) \quad(p \in \mathbb{R}) .
\end{aligned}
$$

For condition (b) it is required $\mathrm{d}^{3}$ that for all states $f$ and all $(Q, P) \in \mathbb{R}^{2}$

$$
f(q)=0 \quad(|q|>Q) \Rightarrow C_{f}^{(\Phi)}(q, p)=0 \quad(|q|>Q),
$$

and

$$
(\mathscr{F} f)(p)=0 \quad(|p|>P) \Rightarrow C_{f}^{(\Phi)}(q, p)=0 \quad(|p|>P) .
$$

It can be shown ${ }^{3}$ that validity of $(1.11)$ for all $f$ is equivalent to

$$
\int e^{-2 \pi i \theta q} \Phi(\theta, \tau) d \theta=0 \quad(|q|>|\tau| / 2),
$$

for all $\tau \in \mathbb{R}$; similarly, validity of (1.12) for all $f$ is equivalent to

$$
\int e^{-2 \pi i \tau p} \Phi(\theta, \tau) d \tau=0 \quad(|p|>|\theta| / 2),
$$

for all $\theta \in \mathbb{R}$. That is, $\Phi(\cdot, \tau), \Phi(\theta, \cdot)$ are functions of the PaleyWiener kind ${ }^{5}$ with type $\leqslant|\tau| / 2, \leqslant|\theta| / 2$, respectively, for $(\theta, \tau) \in \mathbf{R}^{2}$ when the finite support properties are satisfied.

For property (c) to hold, we must have that Moyal's formula ${ }^{6-8}$

$$
\iint C_{f}^{(\Phi)}(q, p) \overline{C_{g}^{(\Phi)}(q, p)} d q d p=|(f, g)|^{2}
$$

is valid for all states $f$ and $g$. It has been shown ${ }^{9}$ that validity of (1.15) for all $f$ and $g$ is equivalent to

$$
|\Phi(\theta, \tau)|=1 \quad\left[(\theta, \tau) \in \mathbb{R}^{2}\right],
$$

or

$$
(\varphi * \tilde{\varphi})(q, p)=\delta(q) \delta(p) \quad\left[(q, p) \in \mathbb{R}^{2}\right],
$$

where $\tilde{\varphi}(q, p)=\overline{\varphi(-q,-p)}$, and *denotes convolution over $\mathbf{R}^{2}$. A further result ${ }^{9}$ is that validity of $(1.15)$ for all $f$ and $g$, together with validity of (1.7), (1.11), and (1.12) for all $f$, implies that $\Phi$ takes the special form

$$
\Phi(\theta, \tau)=\Phi_{\alpha}(\theta, \tau)=\exp (2 \pi i \alpha \theta \tau) \quad\left[(\theta, \tau) \in \mathbb{R}^{2}\right]
$$

for some $\alpha \in \mathbf{R}$ with $|\alpha| \leqslant \frac{1}{2}$. In that case $\varphi$ is given by

$$
\varphi(q, p)=\varphi_{\alpha}(q, p)=\alpha^{-1} \exp (-2 \pi i q p / \alpha) \text { or } \delta(q) \delta(p)
$$

$\left[(q, p) \in \mathbb{R}^{2}\right]$

according as $\alpha \neq 0$ or $\alpha=0$, and $C_{f}^{(\Phi)}$ takes the special form ${ }^{9}$

$$
\begin{aligned}
C_{f}^{(\Phi)}(q, p)= & C_{f}^{\left(\Phi_{\alpha}\right)}(q, p) \\
= & \int e^{-2 \pi i p t} f\left(q+t\left(\frac{1}{2}-\alpha\right)\right) \\
& \times f \overline{\left(q-t\left(\frac{1}{2}+\alpha\right)\right)} d t\left[(q, p) \in \mathbb{R}^{2}\right] .
\end{aligned}
$$

It is interesting to note that for any state $f$ and any $(a, b) \in \mathbf{R}^{2}$ the global spread

$$
\iint\left[(q-a)^{2}+(p-b)^{2}\right]\left|C_{f}^{\left(\Phi_{\alpha}\right)}(q, p)\right|^{2} d q d p
$$

of $C_{f}^{\left(\phi_{\alpha}\right)}$ around $(a, b)$ is minimal for $\alpha=0$, the Wigner distribution case. Choosing for $(a, b)$ the center of gravity ${ }^{8}$ of $C_{f}^{\left(\Phi_{\alpha}\right)}$, which is independent of $\alpha$ and equals ${ }^{9}$

$$
(a, b)=\left(\int q|f(q)|^{2} d q, \int p|(\mathscr{F} f)(p)|^{2} d p\right),
$$

we see that the Wigner distribution behaves, in some sense, best with respect to spread among the members of Cohen's class that satisfy conditions (a), (b), and (c). This is some indication that the Wigner distribution is to be preferred over the other members of Cohen's class. One may find this argument not entirely convincing yet, for one has to restrict oneself to distributions satisfying the strong condition that Moyal's formula is satisfied and this excludes, for example, the family of distributions ( $f$ arbitrary state)

$$
\operatorname{Re}\left[e^{2 \pi i q p} \overline{f(q)}\left(\mathscr{F}_{f}\right)(p)\right] \quad\left[(q, p) \in \mathbb{R}^{2}\right],
$$

which was considered by Margenau and Hill. ${ }^{10}$

We finally discuss condition (d). This condition says that for all $f$ it should hold that ${ }^{11}$

$$
C_{f}^{(\Phi)}(q, p) \geqslant 0 \quad\left[(q, p) \in \mathbb{R}^{2}\right] .
$$

It has been shown ${ }^{12}$ that validity of (1.7) and (1.24) for all states $f$ is not possible. This does not contradict the result of Ref. 13 where to every state a non-negative function of $(q, p)$ with correct marginal distributions is assigned in a nonbilinear way.

With respect to positivity properties only the Wigner distribution has been studied in some detail ${ }^{14-16}$ as far as we know. It is exactly the purpose of this paper to compare the general phase-plane distribution functions on this point with the Wigner distribution. The best known positivity property of the Wigner distribution ${ }^{17-21}$ reads: for all states $f$, all $\gamma>0$, $\delta>0$ with $\gamma \delta \leqslant 1$, and all $(a, b) \in \mathbb{R}^{2}$ we have

$\iint \exp \left[-2 \pi \gamma(q-a)^{2}-2 \pi \delta(p-b)^{2}\right] W_{f}(q, p) d q d p \geqslant 0$.

This paper concentrates on finding out for what $\Phi$ and what $\gamma, \delta$ inequality $(1.25)$ still holds for all $f,(a, b)$ when $W_{f}$ is replaced by the more general phase-plane distribution $C_{f}^{(\Phi)}$. In connection with $(1.25)$ we note that the following has been proved for the Wigner distribution. Hudson ${ }^{17}$ has shown that $W_{f}$ takes negative values unless $f$ is a Gaussian. The argument used by Hudson was augmented ${ }^{21}$ to show that, if $\gamma \delta>1$, any $f$ for which (1.25) is non-negative for all $(a, b) \in \mathbb{R}^{2}$ must be a (possibly degenerate) Gaussian (in Ref. 21 certain generalized functions are allowed; we turn to these in Sec. II). It is not clear how a result of similar strength can be shown to hold generally for the distributions of Cohen. We have, e.g., with $\Phi(\theta, \tau)=\cos \pi \theta \tau$ [which yields (1.23)] that $C_{f}^{(\phi)}(q, p) \geqslant 0$ for $f(q)=\cos 2 \pi q$. Nevertheless the following results will be proved in this paper. Assume that $\Phi$ is such that (1.7) is satisfied for all $f$. Under a mild smoothness and growth condition ${ }^{22}$ on $\Phi$ we have the following.

(1) If $\gamma \delta>1$, then there is no $\Phi$ such that (1.25) (with $C_{f}^{(\Phi)}$ instead of $\left.W_{f}\right)$ holds for all $f$ and all $(a, b) \in \mathbb{R}^{2}$.

(2) If $\gamma \delta=1$, then the only $\Phi$ for which (1.25) (with $C_{f}^{(\Phi)}$ instead of $\left.W_{f}\right)$ holds for all $f$ and all $(a, b) \in \mathbb{R}^{2}$ equals $\Phi(\theta, \tau)=1$ (Wigner distribution case). 
We shall prove that a similar result holds when validity of (1.7) is replaced by validity of $(1.15)$ for all $f$ and $g$. We shall in addition show that validity of $(1.25)$ (with $C_{f}^{(\Phi)}$ instead of $W_{f}$ ) imposes severe restrictions on $\Phi$ if $\gamma \delta<1$ and (1.7) is satisfied for all $f$, or if $\gamma \delta \geqslant 1$.

The further plan of this paper is as follows. In Sec. II we give a mathematical setting that allows us to consider functions $\Phi$ with mild restrictions on growth. We furthermore recall in Sec. II the main results of Ref. 16, and we extend these results somewhat. In Ref. 16 conditions for a function $K(q, p)$ are given that ensure that

$$
\iint K(q, p) W_{f}(q, p) d q d p
$$

is non-negative for all $f$. It is clear that these results will be useful, since (1.5) and (1.25) show that non-negativity of (1.25) [with $C_{f}^{(\Phi)}$ instead of $W_{f}$ and $\left.(a, b)=(0,0)\right]$ for all $f$ is equivalent to non-negativity of $(1.26)$ for all $f$, where $K$ is the convolution of $\varphi(q, p)$ and $\exp \left(-2 \pi \gamma q^{2}-2 \pi \delta p^{2}\right)$. In Sec. III we consider the case that no other condition than nonnegativity of $(1.25)$ [with $C_{f}^{(\phi)}$ instead of $W_{f}$ and $(a, b)=(0,0)$ ] for all $f$ is imposed; in Sec. IV we require in addition correct marginals or validity of Moyal's formula.

\section{MATHEMATICAL SETTING AND RESULTS ON POSITIVITY FOR THE WIGNER DISTRIBUTION}

As we have to discuss rather general functions $\Phi$ it is convenient to restrict the states $f$ to a certain space of test functions. We consider the space $S$ of smooth functions; this function space has been proposed in Ref. 8 as a setting suited for doing Wigner distribution analysis. It is the same space as the one used in Refs. 16,21, and 23 . To describe it briefly we denote, for $n=0,1, \ldots$, by $\psi_{n}$ the $n$th Hermite function,

$$
\psi_{n}(q)=\frac{(-1)^{n} 2^{1 / 4} e^{\pi q^{2}}(d / d q)^{n} e^{-2 \pi q^{2}}}{n !(4 \pi)^{n / 2}} \quad(q \in \mathbb{R}) ;
$$

the normalization has been chosen in such a way that

$e^{\pi q^{2}-2 \pi(q-w)^{2}}=2^{-1 / 4} \sum_{n=0}^{\infty} \frac{(2 w \sqrt{\pi})^{n}}{\sqrt{n !}} \psi_{n}(q) \quad(q \in \mathbb{R}, w \in \mathbb{C})$.

The space $S$ consists of all functions $f$ whose Hermite coefficients $\left(f, \psi_{n}\right)$ satisfy an estimate

$$
\left(f, \psi_{n}\right)=O\left(e^{-n \alpha}\right) \quad(n=0,1, \ldots),
$$

for some $\alpha>0$. It can be shown that the space $S$ is identical to the set of (restrictions to the real axis of) entire functions $g$ for which there are $M>0, A>0, B>0$ such that

$$
|g(x+i y)| \leqslant M \exp \left(-\pi A x^{2}+\pi B y^{2}\right) \quad\left[(x, y) \in \mathbb{R}^{2}\right] .
$$

A sequence $\left(f_{k}\right)_{k}$ in $S$ is said to converge to zero when, for some $\alpha>0, \sup _{n=0,1, \ldots} e^{n \alpha}\left|\left(f_{k}, \psi_{n}\right)\right| \rightarrow 0$ when $k \rightarrow \infty$.

The space $S^{*}$ consists of all continuous linear functionals on $S$. It can be shown that for $F \in S^{*}$

$$
\left(F, \psi_{n}\right)=O\left(e^{n \alpha}\right) \quad(n=0,1, \ldots),
$$

for all $\alpha>0$. The smoothing operators $N_{\alpha}$ with $\operatorname{Re} \alpha>0$ play an important role; they map $S^{*}$ into $S$ and are defined by

$$
\left(N_{\alpha} F\right)(q)=\sum_{n=0}^{\infty}\left(F, \psi_{n}\right) e^{-(n+1 / 2) \alpha} \psi_{n}(q) \quad\left(F \in S^{*}, q \in \mathbb{C}\right) .
$$

As an integral operator of $L^{2}(\mathbb{R}), N_{\alpha}$ has the kernel $K_{\alpha}$ given by

$$
\begin{aligned}
& K_{\alpha}(q, p)=\left(\frac{1}{\sinh \alpha}\right)^{1 / 2} \exp \left(-\frac{\pi}{\sinh \alpha}\right. \\
&\left.\times\left[\left(q^{2}+p^{2}\right) \cosh \alpha-2 q p\right]\right) \\
&= \sum_{n=0}^{\infty} e^{-(n+1 / 2) \alpha} \psi_{n}(q) \psi_{n}(p) \\
& {\left[(q, p) \in \mathbb{R}^{2}\right] . }
\end{aligned}
$$

The identity in (2.7) is just one way to write Mehler's formula

$$
\begin{gathered}
\left(\frac{2}{1-w^{2}}\right)^{1 / 2} \exp \left(-\pi\left(q^{2}+p^{2}\right) \frac{1+w^{2}}{1-w^{2}}+4 \pi \frac{q p w}{1-w^{2}}\right) \\
=\sum_{n=0}^{\infty} w^{n} \psi_{n}(q) \psi_{n}(p) \quad\left[(q, p) \in \mathbb{C}^{2},|w|<1\right] .
\end{gathered}
$$

The spaces $S^{2}$ and $S^{2 *}$ of smooth and generalized functions of two variables can be defined in a similar fashion. An important formula, relating smoothing operators and Wigner distributions, ${ }^{24}$ reads

$$
\left(N_{\alpha, 2} V_{f}\right)(q, p)=V_{N_{o} f}(q, p) \quad\left[(q, p) \in \mathbb{R}^{2}, \operatorname{Re} \alpha>0\right]
$$

for $f \in L^{2}(\mathbb{R})$. Here $N_{\alpha, 2}$ is the smoothing operator for functions of two variables [whose kernel $K_{\alpha, 2}(q, p ; x, y)$ equals $\left.K_{\alpha}(q, x) K_{\alpha}(p, y)\right]$, and

$$
V_{f}(q, p)=\frac{1}{\sqrt{2}} W_{f}\left(\frac{q}{\sqrt{2}}, \frac{p}{\sqrt{2}}\right) \quad\left[(q, p) \in \mathbb{R}^{2}\right]
$$

for $f \in L^{2}(\mathbb{R})$. We note ${ }^{25}$ that $V_{F}$ (and hence $W_{F}$ ) can be defined for $F \in S^{*}$ and that $V_{F} \in S^{2 *}$.

Another useful formula ${ }^{26}$ is

$$
\begin{aligned}
& W_{N_{i f} f}(q, p)=W_{f}(q \cos \theta+p \sin \theta, p \cos \theta-q \sin \theta) \\
& {\left[(q, p) \in \mathbb{R}^{2}\right],}
\end{aligned}
$$

which holds for all real $\theta$ and all $f \in S$.

In spite of the rather heavy machinery we have developed here, we shall usually manipulate with generalized functions in a rather carefree manner; we shall give details only in cases where the verification are not straightforward.

We now turn to positivity properties of the Wigner distribution. We have, for $n=0,1, \ldots,{ }^{27}$

$$
\begin{aligned}
W_{\psi_{n}}(q, p)= & 2(-1)^{n} \exp \left[-2 \pi\left(q^{2}+p^{2}\right)\right] \\
& \times L_{n}\left[4 \pi\left(q^{2}+p^{2}\right)\right] \quad\left[(q, p) \in \mathbb{R}^{2}\right] .
\end{aligned}
$$

Here $L_{n}$ is the $n$th Laguerre polynomial,

$$
L_{n}(x)=\sum_{j=0}^{n}\left(\begin{array}{l}
n \\
j
\end{array}\right) \frac{(-x)^{j}}{j !} \quad(x \geqslant 0 ; n=0,1, \ldots),
$$

for which a generating formula ${ }^{28}$ is given by

$$
\begin{aligned}
& (1-w)^{-1} \exp \left[-x w(1-w)^{-1}\right] \\
& =\sum_{n=0}^{\infty} w^{n} L_{n}(x) \quad(|w|<1, x \geqslant 0) .
\end{aligned}
$$

Formula (2.12) can be used to show the identity ${ }^{29}$

$$
\begin{aligned}
\iint W_{f}(q, p) K\left[2 \pi\left(q^{2}+p^{2}\right)\right] d q d p \\
=\sum_{n=0}^{\infty}(-1)^{n}\left|\left(f, \psi_{n}\right)\right|^{2} \int_{0}^{\infty} e^{-r} K(r) L_{n}(2 r) d r
\end{aligned}
$$


where $f \in S$ and $K:[0, \infty) \rightarrow \mathbb{C}$ is measurable and satisfies

$$
\int_{0}^{\infty}|K(x)|^{2} e^{-\epsilon x} d x<\infty \quad(\epsilon>0) .
$$

Now positivity properties of the Wigner distribution result on taking non-negative functions $K$ with the property that

$$
(-1)^{n} \int_{0}^{\infty} e^{-r} K(r) L_{n}(2 r) d r \geqslant 0 \quad(n=0,1, \ldots) .
$$

In Ref. 16 a large number of examples of such $K$ 's have been given. We mention in particular the choices

$$
\begin{aligned}
& K(r)=r^{n} e^{-\rho r} \quad(0 \leqslant \rho \leqslant 1, \quad n=0,1, \ldots), \\
& K(r)=r^{\alpha} \quad\left(\alpha \geqslant-\frac{1}{2}\right) .
\end{aligned}
$$
know.

The following positivity property is new as far as we

Theorem 2.1: Let $K:[0, \infty) \rightarrow[0, \infty)$ be nondecreasing, and assume that $K(x)=O[\exp (\epsilon x)]$ for some $\epsilon<1$. Then (2.17) holds.

Proof: It follows from Bonnet's theorem ${ }^{30}$ that for all $A>0$ there is an $x_{0}(A) \in[0, A]$ such that

$$
\begin{aligned}
& (-1)^{n} \int_{0}^{A} e^{-r} L_{n}(2 r) K(r) d r \\
& =(-1)^{n} K(A-) \int_{x_{0}(A)}^{A} e^{-r} L_{n}(2 r) d r
\end{aligned}
$$

It is easy to check from formula (2.14) that

$$
(-1)^{n} \int_{r}^{\infty} e^{-s} L_{n}(2 s) d s=S_{n}(r)+S_{n-1}(r) \quad(r \geqslant 0)
$$

where

$$
S_{n}(r)=\sum_{k=0}^{n}(-1)^{k} e^{-r} L_{k}(2 r) \quad(n \geqslant-1, r \geqslant 0) .
$$

Since in Ref. 28, Problem 100, p. 392, shows that $S_{n}(r) \geqslant 0$ for $n \geqslant-1, r \geqslant 0$, it follows that

$$
\begin{aligned}
& (-1)^{n} K(A-) \int_{\mathrm{x}_{0}(A)}^{\infty} e^{-r} L_{n}(2 r) d r \geqslant 0 \\
& (A \geqslant 0, n=0,1, \ldots) .
\end{aligned}
$$

The proof is easily completed by noting that, for $n=0,1, \ldots$,

$$
K(A-) \int_{A}^{\infty} e^{-r} L_{n}(2 r) d r \rightarrow 0 \quad(A \rightarrow \infty) .
$$

Notes: (1) Assume that $K$ is infinitely many times differentiable, and that $K(r)$ and all its derivatives are $O\left(e^{\epsilon r}\right)$ for some $\epsilon<1$. Then (2.17) holds if and only if

$$
\int_{0}^{\infty} r^{n} e^{-r}\left(\frac{d}{d r}\right)^{n}\left[e^{r / 2} K\left(\frac{r}{2}\right)\right] d r \geqslant 0 \quad(n=0,1, \ldots)
$$

This follows on using $e^{-r} L_{n}(r)=1 / n !(d / d r)^{n}\left(e^{-r^{r} r^{n}}\right)$ and performing $n$ partial integrations in (2.17).

(2) Since both $K(r)=r^{\alpha}\left(\alpha \geqslant-\frac{1}{2}\right)$ and

$K(r)=e^{-\rho r}(0 \leqslant \rho \leqslant 1)$ satisfy (2.17), one may ask whether $K(r)=r^{\alpha} e^{-\rho r}$ satisfies (2.17). Well, it does not unless $\alpha$ is an integer. It can be shown from the formula (2.14) that, for $n=0,1, \ldots$,

$$
\begin{aligned}
(-1)^{n} \int_{0}^{\infty} e^{-r} L_{n}(2 r) r^{\alpha} e^{-\rho r} d r \\
=(1-\rho)^{-\alpha-1} \Gamma(\alpha+1) C_{w^{n}}\left[(1+w)^{\alpha}\right. \\
\left.\quad \times\left(\frac{1+\rho}{1-\rho}-w\right)^{-\alpha-1}\right] .
\end{aligned}
$$

Here $C_{w^{n}}$ denotes "coefficient of $w^{n}$ in." Now Darboux's method $^{31}$ can be used to find the asymptotic behavior of the coefficients of the function $(1+w)^{\alpha}[(1+\rho) /(1-\rho)$

$$
-w]^{-\alpha-1} \text {. We get }[a=(1+\rho) /(1-\rho)]
$$

$$
\begin{aligned}
&(-1)^{n} \int_{0}^{\infty} e^{-r} L_{n}(2 r) r^{\alpha} e^{-\rho r} d r \\
&= \frac{\alpha(\alpha-1) \cdots(\alpha-n+1)}{n !} \\
& \times\left[1+\frac{(\alpha+1)^{2}}{(a+1)(\alpha-n+1)}+O\left(\frac{1}{n^{2}}\right)\right] \\
&(n=0,1, \ldots),
\end{aligned}
$$

and this oscillates for large $n$ when $\alpha$ is noninteger. This example shows that the condition (2.17) is rather intricate.

(3) We give an application of formula (2.15) which has nothing to do with the main subject of this paper. In the context of the Weyl quantization map we can express the left-hand side of $(2.15)$ as $\left(T_{K} f f\right)$, where $T_{K}$ is the linear operator whose Weyl symbol ${ }^{32}$ equals $K\left[2 \pi\left(q^{2}+p^{2}\right)\right]$. Denote by $H$ the Hermite operator $-\left(1 / 4 \pi^{2}\right)\left(d^{2} / d q^{2}\right)+q^{2}$, whose Weyl symbol equals $q^{2}+p^{2}$. One can now ask how well $f\left(q^{2}+p^{2}\right)$ is an approximation to the Weyl symbol of $f(H)$. As an example we consider $f(r)=r^{1 / 2}$, and to that end we choose $K(r)=(r / 2 \pi)^{1 / 2}$ in $(2.17)$. Now $T_{K}$ is an operator whose matrix relative to the basis $\left(\psi_{n}\right)_{n=0,1, \ldots}$ of Hermite functions is a diagonal matrix, with diagonal elements

$$
\begin{aligned}
\left(T_{K} \psi_{n}, \psi_{n}\right) & =\frac{(-1)^{n}}{\sqrt{2 \pi}} \int_{0}^{\infty} e^{-r} L_{n}(2 r) r^{1 / 2} d r \\
& =2^{-3 / 2} C_{w^{n}}\left[(1-w)^{1 / 2} /(1+w)^{1 / 2}\right] .
\end{aligned}
$$

By using Darboux's method, one can show that

$$
\begin{aligned}
& \left(T_{K} \psi_{n}, \psi_{n}\right)=\pi^{-1 / 2}\left(n+\frac{1}{2}\right)^{1 / 2}[1+O(1 / n)] \\
& (n=0,1, \ldots) .
\end{aligned}
$$

At the same time $\left(\sqrt{H} \psi_{n}, \psi_{n}\right)=\pi^{-1 / 2}\left(n+\frac{1}{2}\right)^{1 / 2}$ for $n=0,1, \ldots$. Hence $T_{K}-\sqrt{H}$ is a diagonal operator (relative to the $\psi_{n}$ 's) with diagonal elements that are $O\left(n^{-1 / 2}\right)$. This shows that $T_{K}-\sqrt{H}$ is of Schatten's $p$ class with $p>2$. Of course, all sorts of generalizations are possible here.

\section{PHASE-PLANE DISTRIBUTION FUNCTIONS WITH NON-NEGATIVE GAUSSIAN AVERAGES}

Let $\gamma>0$. In this section we want to find out for which $\Phi$ as in (1.1) or $\varphi$ as in (1.6) we have

$$
\iint C_{f}^{(\Phi)}(q, p) \exp \left[-2 \pi \gamma\left(q^{2}+p^{2}\right)\right] d q d p \geqslant 0
$$

for all $f \in S$. We require here that $\Phi \in S^{2 *}$ or $\varphi \in S^{2 *}$, for then formula (1.5) shows that $C_{f}^{(\Phi)}$ is the convolution of $\varphi \in S^{2 *}$ 
and $W_{f} \in S^{2}$, and this is a smooth function that can be integrated against any Gaussian as in (3.1). For the details concerning convolution theory in the spaces $S, S^{2}, S^{*}, S^{2 *}$, one may consult Ref. 33 . We consider here only radially symmetric Gaussian weight functions since the more general Gaussians $\exp \left[-2 \pi\left(\gamma q^{2}+\delta p^{2}\right)\right]$ can be dealt with by considering $\Phi\left(\alpha^{-1} \theta, \alpha \tau\right)$ instead of $\Phi(\theta, \tau)\left[\alpha=(\delta / \gamma)^{1 / 2}\right]$. Wecan write (3.1) as

$$
\iint G(a, b) W_{f}(a, b) d a d b
$$

with $G$ the convolution of $\varphi$ and $\exp \left[-2 \pi \gamma\left(q^{2}+p^{2}\right)\right]$, i.e.,

$$
\begin{aligned}
G(a, b)= & \iint \varphi(q-a, p-b) \\
& \times \exp \left[-2 \pi \gamma\left(q^{2}+p^{2}\right)\right] d q d p \quad\left[(a, b) \in \mathbb{R}^{2}\right] .
\end{aligned}
$$

The following results show that a $G$ for which (3.2) is non-negative for all $f \in S$ cannot decay too rapidly.

Lemma 3.1: Assume that $G: \mathbb{R}^{2} \rightarrow \mathbb{R}$ is bounded and measurable and satisfies $G(a, b)=o\left(\exp \left[-2 \pi\left(a^{2}+b^{2}\right)\right]\right)$ $\left(a^{2}+b^{2} \rightarrow \infty\right)$. Then (3.2) is negative for some $f \in S$, unless

$$
\int_{0}^{2 \pi} G(R \cos \theta, R \sin \theta) d \theta=0 \quad(R \geqslant 0)
$$

Proof: Part of the argument given here can also be found in Ref. 16. Suppose that (3.2) is non-negative for all $f \in S$, and let

$$
K(r)=\frac{1}{2 \pi} \int_{0}^{2 \pi} G\left(\sqrt{\frac{r}{2 \pi}} \cos \theta, \sqrt{\frac{r}{2 \pi}} \sin \theta\right) d \theta .
$$

We have for any $f \in S$ by (2.11)

$$
\begin{aligned}
\iint K & {\left[2 \pi\left(q^{2}+p^{2}\right)\right] W_{f}(q, p) d q d p } \\
= & \frac{1}{2 \pi} \int_{0}^{2 \pi}\left(\iint G(q, p) W_{N_{i \theta f}}(q, p) d q d p\right) d \theta \geqslant 0 .
\end{aligned}
$$

Therefore, by (2.15), we have, for all $n$,

$$
a_{n}:=(-1)^{n} \int_{0}^{\infty} e^{-r} K(r) L_{n}(2 r) d r \geqslant 0 .
$$

It follows from the formula ${ }^{34}$

$$
\begin{gathered}
r^{\alpha}=2^{-\alpha} \sum_{n=0}^{\infty}(-1)^{n} \frac{\Gamma^{2}(\alpha+1)}{n ! \Gamma(\alpha-n+1)} L_{n}(2 r) \\
(\alpha>-1, r>0)
\end{gathered}
$$

that

$$
\int_{0}^{\infty} r^{\alpha} e^{-r} K(r) d r=2^{-\alpha} \sum_{n=0}^{\infty} \frac{\Gamma^{2}(\alpha+1)}{n ! \Gamma(\alpha-n+1)} a_{n} .
$$

The left-hand side of (3.9) can be shown to be $o\left[2^{-\alpha} \Gamma(\alpha+1)\right]$ as $\alpha \rightarrow \infty$. Indeed, this follows from the assumptions on $G$ implying that $K(r)=o\left(e^{-\eta}\right)$ as $r \rightarrow \infty$. The sum on the right-hand side of (3.9) has, for integer $\alpha$, nonnegative terms only. Hence, for any $m=0,1, \ldots$, we have

$$
\begin{aligned}
& 2^{-\alpha} \sum_{n=0}^{\infty} \frac{\Gamma^{2}(\alpha+1)}{n ! \Gamma(\alpha-n+1)} a_{n} \geqslant 2^{-\alpha} \frac{\Gamma^{2}(\alpha+1)}{m ! \Gamma(\alpha-m+1)} a_{m} \\
&= 2^{-\alpha} \Gamma(\alpha+1)(\alpha-m+1) \cdots(\alpha+1) a_{m} / m ! \\
&(\alpha=m, m+1, \ldots) .
\end{aligned}
$$

This is certainly not $o\left[2^{-\alpha} \Gamma(\alpha+1)\right]$ as $\alpha \rightarrow \infty$, unless all $a_{m}$ are 0 . Since the functions $e^{-r} L_{n}(2 r), n=0,1, \ldots$ are complete in $L^{2}([0, \infty))$, we see that $K=0$, and the proof is finished.

Note: With a similar proof one can show that if $G$ is radially symmetric and satisfies

$G(a, b)=O\left(\left(a^{2}+b^{2}\right)^{p} \exp \left[-2 \pi\left(a^{2}+b^{2}\right]\right) \in \mathbb{R}^{2}\right.$,

for some $p \geqslant 0$, and (3.2) is non-negative for all $f \in S$, then $G$ is of the form

$$
\begin{aligned}
G(a, b)= & \sum_{n<p}(-1)^{n} a_{n} \exp \left[-2 \pi\left(a^{2}+b^{2}\right)\right] \\
& \left.\times L_{n}\left[4 \pi\left(a^{2}+b^{2}\right)\right] \quad[a, b) \in \mathbb{R}^{2}\right],
\end{aligned}
$$

with $a_{n} \geqslant 0(n \leqslant p)$.

Theorem 3.1: Assume that $G: \mathbb{R}^{2} \rightarrow \mathbb{R}$ is continuous and that

$$
G(a, b)=O\left(\exp \left[-2 \pi \delta\left(a^{2}+b^{2}\right)\right]\right) \quad\left[(a, b) \in \mathbb{R}^{2}\right],
$$

for some $\delta>1$. If (3.2) is non-negative for all $f \in S$, then $G=0$.

Proof: Let $\left(a_{0}, b_{0}\right) \in \mathbb{R}^{2}$, and let

$$
G_{0}(a, b):=G\left(a-a_{0}, b-b_{0}\right) \quad\left[(a, b) \in \mathbb{R}^{2}\right] .
$$

We see from (1.2) that (3.2) holds for all $f$ (with $G_{0}$ instead of $G)$. Furthermore

$$
G_{0}(a, b)=O\left(\exp \left[-2 \pi \epsilon\left(a^{2}+b^{2}\right)\right]\right) \quad\left[(a, b) \in \mathbb{R}^{2}\right],
$$

for any $\epsilon$ between 1 and $\delta$. Now Lemma 3.1 shows that

$$
\int_{0}^{2 \pi} G_{0}(R \cos \theta, R \sin \theta) d \theta=0 \quad(R \geqslant 0) .
$$

It then follows from continuity of $G$ that $G_{0}(0,0)=G\left(a_{0}, b_{0}\right)=0$. This completes the proof.

Note: It is clear that the conditions on $G$ can be weakened somewhat.

Theorem 3.2: Let $\gamma>1$ and let $\delta>\gamma(\gamma-1)^{-1}$. Assume that $\varphi: \mathbb{R}^{2} \rightarrow \mathbb{R}$ satisfies

$$
\varphi(q, p)=O\left(\exp \left[-2 \pi \delta\left(q^{2}+p^{2}\right)\right]\right) \quad\left[(q, p) \in \mathbb{R}^{2}\right] .
$$

Then there is an $f \in S$ for which (3.1) is negative, unless $\varphi=0$. In particular, there is no compactly supported $\varphi \neq 0$ such that (3.1) is non-negative for all $f \in S$.

Proof: Let $G$ be as in (3.3). Then $G$ is smooth and satisfies

$G(a, b)=O\left[\exp \left(-2 \pi \frac{\delta \gamma}{\delta+\gamma}\left(a^{2}+b^{2}\right)\right)\right] \quad\left[(a, b) \in \mathbb{R}^{2}\right]$.

As $\delta \gamma /(\delta+\gamma)>1$, the theorem follows from Theorem 3.1.

Note: We can allow $\varphi$ to be an element of $S^{2 *}$ if we have a substitute for condition (3.17). The theorem also holds, for instance, when $N_{\alpha, 2} \varphi$ (instead of $\varphi$ ) satisfies (3.17) for some $\alpha>0$. This is a consequence of (2.9). The theorem also holds 
when one requires that $\Phi$ be an entire function of two variables with

$$
\Phi(\theta, \tau)=O\left[\exp \left(\frac{\pi \epsilon}{2}\left(|\theta|^{2}+|\tau|^{2}\right)\right)\right] \quad\left[\left(\theta, \tau \mid \in \mathbb{C}^{2}\right]\right.
$$

for some $\epsilon<(\gamma-1) / \gamma$, for then the $G$ of (3.3) also satisfies (3.13) with a $\delta>1$. All these matters can be proved rigorously within the framework of the theory in Ref. 33 .

Example: Let $\gamma>0$ and consider the choice $\Phi_{0}(\theta, \tau)=\exp (2 \pi i \alpha \theta \tau)$ with $\alpha \in \mathbb{R}, \alpha \neq 0$. Now $\varphi_{0}$ is given by

$$
\varphi_{0}(q, p)=\alpha^{-1} \exp \left(-2 \pi i \alpha^{-1} q p\right) \quad\left[(q, p) \in \mathbb{R}^{2}\right],
$$
and the $G=G_{0}$ of (3.3) can be shown to equal

$$
\begin{aligned}
G_{0}(a, b)= & \left(1+4 \gamma^{2} \alpha^{2}\right)^{-1 / 2} \\
& \times \exp \left(-\frac{2 \pi \gamma\left(a^{2}+b^{2}\right)}{1+4 \gamma^{2} \alpha^{2}}-\frac{8 \pi i \alpha \gamma^{2} a b}{1+4 \gamma^{2} \alpha^{2}}\right)
\end{aligned}
$$

$\left[(a, b) \in \mathbb{R}^{2}\right]$.

Let $g$ be the Gaussian $2^{1 / 4} \exp \left[-\pi(1+i) q^{2}\right]$ whose Wigner distribution equals

$$
W_{g}(q, p)=2 \exp \left(-2 \pi\left[q^{2}+(q+p)^{2}\right]\right) \quad\left[(q, p) \in \mathbb{R}^{2}\right] .
$$

The convolution of $W_{g}$ and $G_{0}$ is a function of the form

$$
\begin{aligned}
& \left(W_{g} * G_{0}\right)(q, p) \\
& \quad=\exp \left[-\pi P_{1}(q, p)+\pi i P_{2}(q, p)\right] \quad\left[(q, p) \in \mathbb{R}^{2}\right],
\end{aligned}
$$

with $P_{1}$ a positive definite quadratic and $P_{2}$ a real nonconstant quadratic. Letting $\varphi(q, p)=\operatorname{Re}\left[\varphi_{0}(q, p)\right]$ $=\alpha^{-1} \cos 2 \pi \alpha^{-1} q p$, so that $G(a, b)=\operatorname{Re}\left[G_{0}(a, b)\right]$ and $\Phi(\theta, \tau)=\cos 2 \pi \alpha \theta \tau$, we get an example of a $\Phi$ such that (3.2) takes negative values for certain $f$ 's. This is so since the real part of (3.23) does so. Note that this example works for any $\gamma>0$ while Theorem 3.1 and (3.21) predict trouble only for $\gamma /\left(1+4 \gamma^{2} \alpha^{2}\right)>1$.

We consider the case $\gamma=1$, which has our prime interest, in some more detail. The next theorem shows that a $\varphi$ yielding non-negative averages in (3.1) must be of positive type in a certain weak sense.

Theorem 3.3: Assume that $\varphi: \mathbb{R}^{2} \rightarrow \mathbb{R}$ satisfies

$$
\varphi(q, p)=O\left(\exp \left[\pi \epsilon\left(q^{2}+p^{2}\right)\right]\right) \quad\left[(q, p) \in \mathbb{R}^{2}\right],
$$

for all $\epsilon>0$. A necessary condition that (3.1) with $\gamma=1$ is non-negative for all $f \in S$ is that

$$
\int_{0}^{\infty} r^{n} e^{-r} \varphi_{a}\left[\left(\frac{r}{\pi}\right)^{1 / 2}\right] d r \geqslant 0 \quad\left(n=0,1, \ldots, a \in \mathbb{R}^{2}\right) .
$$

Here $\varphi_{a}(R)$ is the average of $\varphi$ over the circle of radius $R$ with center $\underline{a}$, i.e.,

$$
\varphi_{a}(R)=\frac{1}{2 \pi} \int_{0}^{2 \pi} \varphi[\underline{a}+R(\cos \theta, \sin \theta)] d \theta \quad(R \geqslant 0) .
$$

Proof: Assume that (3.1) is non-negative for all $f \in S$. By (1.2) it is sufficient to consider the case $\underline{a}=\underline{0}$. Insert formula (1.5) into (3.1) and interchange integrals. We get, for all $f \in S$,

$$
\begin{array}{r}
\iint \varphi(a, b)\left(\iint \exp \left[-2 \pi\left(q^{2}+p^{2}\right)\right]\right. \\
\left.\times W_{f}(q-a, p-b) d q d p\right) d a d b .
\end{array}
$$

The expression between the large parentheses equals $\frac{1}{2}\left|\left(f, G_{1}(-a,-b)\right)\right|^{2}$, where for all $(a, b) \in \mathbb{R}^{2}$

$$
\begin{aligned}
G_{1}(- & a,-b)(q) \\
& =2^{1 / 4} \exp \left[-\pi(q+a)^{2}-2 \pi i b q-\pi i a b\right]
\end{aligned}
$$

$(q \in \mathbb{R})$.

This follows from the fact that, for all $(a, b) \in \mathbb{R}^{2}$,

$$
\begin{aligned}
& W_{G_{2}\left(-a_{,}-b\right)}(q, p) \\
& \quad=2 \exp \left[-2 \pi(q+a)^{2}-2 \pi(p+b)^{2}\right]
\end{aligned}
$$

$\left[(q, p) \in \mathbb{R}^{2}\right]$,

and Moyal's formula. The choice $f=\psi_{n}$ gives $^{35}$

$$
\begin{aligned}
& \left|\left(\psi_{n}, G_{1}(-a,-b)\right)\right|^{2} \\
& \quad=\left[\left(a^{2}+b^{2}\right)^{n} / n !\right] \exp \left[-\pi\left(a^{2}+b^{2}\right)\right] \quad\left[(a, b) \in \mathbb{R}^{2}\right] .
\end{aligned}
$$

Hence

$$
\begin{aligned}
\iint \varphi(a, b) \exp \left[-\pi\left(a^{2}+b^{2}\right)\right]\left(a^{2}+b^{2}\right)^{n} d a d b \\
=\frac{1}{4 \pi^{n+2}} \int_{0}^{\infty} r^{n} e^{-r} \\
\quad \times\left[\int_{0}^{2 \pi} \varphi\left(\sqrt{\frac{r}{\pi}}(\cos \theta, \sin \theta)\right) d \theta\right] d r \geqslant 0,
\end{aligned}
$$

for all $n=0,1, \ldots$, and the theorem follows.

Note: Observe that $r^{n} e^{-r \sqrt{2 \pi n} / n ! \text { has its maximum for }}$ $r=n$ and that this maximum tends to 1 as $n \rightarrow \infty$. Also, if $\epsilon>0$, the set of $r$ with $r^{n} e^{-r} \sqrt{2 \pi n} / n ! \geqslant \epsilon$ is an interval around $r=n$ with length of the order $\sqrt{2 n \log \epsilon^{-T}}$.

\section{PHASE-PLANE DISTRIBUTIONS, CORRECT MARGINALS AND MOYAL'S FORMULA}

Let $\gamma>0$. In this section we aim at characterizing all functions $\Phi$ (or $\varphi$ ) as in (1.1) [(or 1.6)] such that (3.1) holds for all $f \in S$ and such that the corresponding phase-plane distribution functions have correct marginals or satisfy Moyal's formula [see (1.7) and (1.15)]. In the case $\gamma \geqslant 1$ we shall show that, under certain mild conditions on $\Phi$, the situation is very simple: for $\gamma>1$ no such $\Phi$ exists, for $\gamma=1$ we must have $\Phi(\theta, \tau)=1$ (correct marginals) or $\Phi(\theta, \tau)=\exp$ $[-2 \pi i(\theta a+\tau b)]$ for some $(a, b) \in \mathbb{R}^{2}$ (Moyal). And in the case where $\gamma<1$ and (1.7) is satisfied for all $f \in S$, we are still able to derive certain properties of $\Phi$.

We start with a lemma.

Lemma 4.1: Let $H \in L^{1}\left(\mathbb{R}^{2}\right) \cap L^{2}\left(\mathbb{R}^{2}\right)$, and assume that

$$
\iint H(q, p) W_{f}(q, p) d q d p \geqslant 0 \quad(f \in S) .
$$

There exists $c_{n} \geqslant 0$ with $\Sigma_{n} c_{n}<\infty$ and orthonormal $f_{n} \in L^{2}(\mathbb{R})$ such that

$$
H(q, p)=\sum_{n} c_{n} W_{f_{n}}(q, p) \quad\left[(q, p) \in \mathbb{R}^{2}\right],
$$

with convergence in the $L^{2}\left(\mathbb{R}^{2}\right)$ sense.

Proof: Let $T$ be the linear operator defined for $K \in L^{2}\left(\mathbb{R}^{2}\right)$ 


$$
\begin{gathered}
(T K)(q, p)=\int e^{-2 \pi i p t} K\left(q+\frac{1}{2} t, q-\frac{1}{2} t\right) d t \\
{\left[(q, p) \in \mathbb{R}^{2}\right] .}
\end{gathered}
$$

This $T$ maps $L^{2}\left(\mathbb{R}^{2}\right)$ unitarily onto $L^{2}\left(\mathbb{R}^{2}\right)$ as can be seen from Moyal's formula. ${ }^{36}$ And, letting $(f \otimes \bar{f})\left(q_{1}, q_{2}\right)$

$=f\left(q_{1}\right) \overline{f\left(q_{2}\right)}$, we have $T(f \otimes \bar{f})=W_{f}$, for all $f \in S$. Hence, if $T^{*}$ is the adjoint of $T$,

$$
\left(T^{*} H_{2} \otimes \bar{f}\right) \geqslant 0 \quad(f \in S)
$$

where (, ) denotes the inner product in $L^{2}\left(\mathbb{R}^{2}\right)$. Formula (4.4) extends toall $f \in L^{2}(\mathbb{R})$ since $T^{*} H \in L^{2}\left(\mathbb{R}^{2}\right)$ and $S$ is dense in $L^{2}(\mathbb{R})$. We conclude that $T^{*} H$ has a representation ${ }^{37}$

$$
\left(T^{*} H\right)\left(q_{1}, q_{2}\right)=\sum_{n} c_{n} f_{n}\left(q_{1}\right) \overline{f_{n}\left(q_{2}\right)} \quad\left[\left(q_{1}, q_{2}\right) \in \mathbb{R}^{2}\right],
$$

with $f_{n} \in L^{2}(\mathbb{R})$ orthonormal, $c_{n} \geqslant 0, \Sigma_{n} c_{n}{ }^{2}<\infty$ and convergence in the $L^{2}\left(\mathbb{R}^{2}\right)$-sense. Taking $T$ at both sides of $(4.5)$ we arrive at

$$
H(q, p)=\sum_{n} c_{n} W_{f_{n}}(q, p) \quad\left[(q, p) \in \mathbb{R}^{2}\right],
$$

with convergence in the $L^{2}\left(\mathbb{R}^{2}\right)$ sense.

We still have to prove that $\Sigma_{n} c_{n}<\infty$. To that end we consider $H_{1}(q, p)=(1 / \sqrt{2}) H(q / \sqrt{2}, p / \sqrt{2})$. We have [see (2.10)]

$$
H_{1}(q, p)=\sum_{n} c_{n} V_{f_{n}}(q, p) \quad\left[(q, p) \in \mathbb{R}^{2}\right] .
$$

Let $\alpha>0$, and apply to both sides of (4.7) the smoothing operator $N_{\alpha, 2}$ (see Sec. I). We get by (2.9)

$$
\left(N_{\alpha, 2} H_{1}\right)(q, p)=\sum_{n} c_{n} V_{N_{\alpha} f_{n}}(q, p) \quad\left[(q, p) \in \mathbb{R}^{2}\right],
$$

with convergence in the $S^{2}$ sense. ${ }^{38}$ If we integrate this identity over all $(q, p) \in \mathbb{R}^{2}$, we obtain by $(1.7)$

$$
\iint\left(N_{\alpha, 2} H_{1}\right)(q, p) d q d p=\sqrt{2} \sum_{n} c_{n}\left\|N_{\alpha} f_{n}\right\|^{2},
$$

where \|\| denotes the $L^{2}(\mathbb{R})$ norm. Now $\left\|N_{\alpha} f_{n}\right\|$ increases to $\left\|f_{n}\right\|=1$ for all $n$ [see (2.6)], and ${ }^{39} N_{\alpha, 2} H_{1} \rightarrow H_{1}$ in the $L^{1}\left(\mathbb{R}^{2}\right)$ sense if $\alpha$ iO since $H \in L^{1}\left(\mathbb{R}^{2}\right)$, and whence $H_{1} \in L^{1}\left(\mathbb{R}^{2}\right)$. We conclude that

$$
\sum_{n} c_{n}=\iint H(q, p) d q d p<\infty,
$$

and this completes the proof.

Note: Since $\left\|f_{n}\right\|=1$, we have $\left|W_{f_{n}}(q, p)\right| \leqslant 2$ for $(q, p) \in \mathbb{R}^{2}$. Hence, the convergence of the series in $(4.2)$ is uniform. Since $W_{f_{n}}$ is continuous for every $n$, we furthermore see that the $H$ of Lemma 4.1 is continuous.

We are now ready to prove the following theorem.

Theorem 4.1: Assume that the $G$ of $(3.3)$ is in $L^{1}\left(\mathbb{R}^{2}\right) \cap L^{2}\left(\mathbb{R}^{2}\right)$, and that (3.1) holds for all $f \in S$. Then, (a) if $\gamma>1, C_{f}^{(\Phi)}$ cannot have correct marginals for all $f \in S$; and $(\mathrm{b})$ if $\gamma=1$, and $C_{f}^{(\Phi)}$ has correct marginals for all $f \in S$, then $\Phi=1$, and $C_{f}^{(\Phi)}$ is the Wigner distribution of $f$ for all $f \in S$.

Proof: Assume that $C_{f}^{(\Phi)}$ has correct marginals for all $f \in S$. This means that

$$
\begin{aligned}
& \int \varphi(q, p) d p=\delta(q) \quad(q \in \mathbb{R}), \\
& \int \varphi(q, p) d q=\delta(p) \quad(p \in \mathbb{R}) .
\end{aligned}
$$

Hence, if we integrate the $G$ of (3.3) over all $b$ and $a$, we get, respectively,

$$
\int G(a, b) d b=\left(\frac{1}{2 \gamma}\right)^{1 / 2} \exp \left(-2 \pi \gamma a^{2}\right) \quad(a \in \mathbb{R})
$$

and

$$
\int G(a, b) d a=\left(\frac{1}{2 \gamma}\right)^{1 / 2} \exp \left(-2 \pi \gamma b^{2}\right) \quad(b \in \mathbb{R}) .
$$

Our $G$ satisfies the conditions of Lemma 4.1 and therefore we have the representation (4.2) for $H=G$. With an argument similar to the one used for proving convergence of $\Sigma_{n} c_{n}$ in Lemma 4.1 we can show that

$$
\sum_{n} c_{n}\left|f_{n}(a)\right|^{2}=\int G(a, b) d b \quad(\text { a.e. } a \in \mathbb{R}),
$$

and

$$
\sum_{n} c_{n} \mid\left(\left.\mathscr{F} f_{n}(b)\right|^{2}=\int G(a, b) d a(\text { a.e. } b \in \mathbb{R}) .\right.
$$

Since all $c_{n} \geqslant 0$, we conclude that, for all $n$ by (4.12) and (4.13),

$$
c_{n}^{1 / 2}\left|f_{n}(a)\right| \leqslant(1 / 2 \gamma)^{1 / 4} \exp \left(-\pi \gamma a^{2}\right) \quad(\text { a.e. } a \in \mathbb{R}),
$$

and

$$
\left.c_{n}^{1 / 2}\left|\left(\mathscr{F} f_{n}\right)(b)\right| \leqslant(1 / 2 \gamma)^{1 / 4} \exp \left(-\pi \gamma b^{2}\right) \quad \text { (a.e. } b \in \mathbb{R}\right) .
$$

As we shall show in Lemma 4.2, the conditions (4.16) and (4.17) are incompatible when $\gamma>1$ (unless $c_{n}=0$ ). This completes the proof for the case $\gamma>1$. When $\gamma=1$, it follows from Lemma 4.2 that every $c_{n}^{1 / 2} f_{n}$ is a multiple of the Gaussian $\exp \left(-\pi a^{2}\right)$. Therefore, $c_{n} \neq 0$ for only one $n$, and it easily follows that

$$
G(a, b)=\exp \left[-2 \pi\left(a^{2}+b^{2}\right)\right] \quad\left[(a, b) \in \mathbb{R}^{2}\right] .
$$

Hence, as $G$ is the convolution of $\varphi$ and $\exp \left[-2 \pi\left(a^{2}+b^{2}\right)\right]$, we get $\varphi(q, p)=\delta(q) \delta(p)$. This completes the proof.

Notes: (1) Since the $G$ of $(3.3)$ is the double inverse Fourier transform of $(1 / 2 \gamma) \Phi(\theta, \tau) \exp \left[-(\pi / 2 \gamma)\left(\theta^{2}+\tau^{2}\right)\right]$ it is clear that one should impose certain conditions on smoothness and growth on $\Phi$ to get $G \in L^{1}\left(\mathbb{R}^{2}\right) \cap L^{2}\left(\mathbb{R}^{2}\right)$. For instance, conditions of type (1.13) and (1.14) guarante $e^{40}$ that $G \in L^{1}\left(\mathbb{R}^{2}\right) \sim L^{2}\left(\mathbb{R}^{2}\right)$.

(2) As the proof shows, the theorem can be proved equally well with the Gaussian $\exp \left[-2 \pi \gamma\left(q^{2}+p^{2}\right)\right]$ in $(3.1)$ replaced by certain smooth functions $K(q, p)$ with $\int K(q, p) d p=O\left[\exp \left(-2 \pi \gamma q^{2}\right)\right]$ and $\int K(q, p) d q=O\left[\exp \left(-2 \pi \gamma p^{2}\right)\right]$

In the next theorem we replace the condition of having correct marginals by the condition that Moyal's formula holds. We restrict the class of allowed $\varphi$ 's a little further since we need some results from Ref. 33 about convolution theory in $S^{2}$ and $S^{2 *}$. Of course, if one chooses a different mathematical setting (e.g., a setting based on Schwartz' theory of tempered distributions), one can still prove a theorem as the one below. 
Theorem 4.2: Assume that ${ }^{41} \Phi(\theta, \tau) \exp \left[-\pi \epsilon\left(\theta^{2}\right.\right.$ $\left.\left.+\tau^{2}\right)\right] \in S^{2}$ for all $\epsilon>0$, and that (3.1) holds for all $f \in S$. Then, (a) if $\gamma>1$, Moyal's formula (1.15) cannot hold for all $f \in S$, $g \in S$; and (b) if $\gamma=1$ and Moyal's formula holds for all $f \in S$, $g \in S$, then there is an $(a, b) \in \mathbb{R}^{2}$ with $C_{f}^{(\Phi)}(q, p)$ $=W_{f}(q-a, p-b)$ for all $f \in S\left[(q, p) \in \mathbb{R}^{2}\right]$.

Proof: Assume that Moyal's formula holds for all $f$ and $g$. Then

$$
|\Phi(\theta, \tau)|=1 \quad\left[(\theta, \tau) \in \mathbb{R}^{2}\right] .
$$

In terms of $\varphi$ this condition can be written as

$$
\begin{aligned}
& \iint \varphi(q+a, p+b) \overline{\varphi(a, b)} d a d b \\
&=(\varphi * \tilde{\varphi})(q, p)=\delta(q) \delta(p) \quad\left[(q, p) \in \mathbb{R}^{2}\right] .
\end{aligned}
$$

Here $\tilde{\varphi}(a, b)=\overline{\varphi(-a,-b)}$ for all $(a, b) \in \mathbb{R}^{2}$, and $*$ denotes the convolution product for (generalized) functions of two variables.

By the definition of $G$ and the representation (4.2) we have, with $K(q, p)=\exp \left[-2 \pi \gamma\left(q^{2}+p^{2}\right)\right]$,

$$
\varphi * K=G=\sum_{n} c_{n} W_{f_{n}} .
$$

It will be demonstrated in Appendix A that $c_{n}=0\left(e^{-n \beta}\right)$ for some $\beta>0$, that $f_{n} \in S$ and that the right-hand series converges in the $S^{2}$ sense to $\varphi * K \in S^{2}$. Taking convolution with $\tilde{\varphi}$ at both sides and interchanging the convolution and summation signs at the right-hand side (this is allowed ${ }^{42}$ ), we get

$$
K=\tilde{\varphi} * \varphi * K=\sum_{n} c_{n} \tilde{\varphi} * W_{f_{n}},
$$

by $(4.20)$ and (4.21).

We now observe that the Fourier transform of $\tilde{\varphi}$ equals $\bar{\Phi}(\theta, \tau)$. Hence, Moyal's formula is valid with $\Phi$ as well as with $\bar{\Phi}$. Since $C_{f}^{(\Phi)}=\tilde{\varphi} * W_{f}$ we have

$$
\begin{aligned}
\iint C_{f}^{(\bar{\Phi})}(q, p) d q d p & =\iint\left(\tilde{\varphi} * W_{f}\right)(q, p) d q d p \\
& =\overline{\Phi(0,0)} \iint W_{f}(q, p) d q d p=d\|f\|^{2},
\end{aligned}
$$

where $d=\overline{\Phi(0,0)}$ is a number of modulus 1 . Hence, if we integrate identity (4.22) over the phase plane, we get by (4.23)

$$
\frac{1}{2 \gamma}=\iint K(q, p) d q d p=d \sum_{n} c_{n}\left\|f_{n}\right\|^{2}=d \sum_{n} c_{n} .
$$

We conclude from $c_{n} \geqslant 0$ (all $n$ ) and $|d|=1$ that $d=1$.

On the other hand, (4.22) provides an expansion of $K$ in a series of orthogonal functions, and we have by Parseval's formula

$$
\frac{1}{4 \gamma}=\iint|K(q, p)|^{2} d q d p=\sum_{n} c_{n}^{2} .
$$

Now, if we let $d_{n}=2 \gamma c_{n}$, then $d_{n} \geqslant 0, \Sigma_{n} d_{n}=1, \Sigma_{n} d_{n}^{2}=\gamma$. This is not possible when $\gamma>1$, whence the case $\gamma>1$ has been dealt with.
We shall give two proofs for the case $\gamma=1$, one directly hereafter, and one in Appendix B. When $\gamma=1$, we see that exactly one $d_{n}$ equals 1 ; the others are 0 . Hence,

$$
\varphi * K=\frac{1}{2} W_{f}
$$

for some $f \in S$ with $\|f\|=1$. Take the double inverse Fourier transform of (4.26). We get the identity

$\left(\mathscr{F}^{(1)^{*}} \mathscr{F}^{(2)^{*}} W_{f}\right)(\theta, \tau)$

$$
=\Phi(\theta, \tau) \exp \left[-(\pi / 2)\left(\theta^{2}+\tau^{2}\right)\right] \quad\left[(\theta, \tau) \in \mathbb{R}^{2}\right] .
$$

The expression at the left-hand side of (4.27) can be written as

$$
\begin{aligned}
\left(\mathscr{F}^{(1)^{*} \mathscr{F}(2)^{*}} W_{f}\right)(\theta, \tau) & =\int e^{2 \pi i \theta g} f\left(q+\frac{1}{2} \tau\right) \overline{f\left(q-\frac{1}{2} \tau\right)} d q \\
& =\operatorname{Amb}_{f}(-\tau,-\theta) \quad\left[(\theta, \tau) \in \mathbb{R}^{2}\right] ;
\end{aligned}
$$

here $\mathrm{Amb}_{f}$ is the ambiguity function of $f$ which is well known in radar analysis. ${ }^{43,44}$ From a result of Ref. 44 the following inequality can be derived for ambiguity functions. If $p=1,2, \ldots$, then for any $g$,

$$
\iint\left|\operatorname{Amb}_{g}(\tau, \theta)\right|^{2 p} d \tau d \theta \leqslant \frac{1}{p}\|g\|^{2 p}
$$

if $p=2,3, \ldots$, the only functions $g$ that never vanish, that are twice differentiable, and that achieve equality in (4.29) are of the form

$$
g(q)=\exp \left(-\pi \alpha q^{2}+2 \pi \beta q-\pi \epsilon\right) \quad(q \in \mathbb{R}),
$$

with arbitrary complex $\alpha, \beta, \epsilon$, and $\operatorname{Re} \alpha>0$.

It is easily verified from the fact that $|\Phi(\theta, \tau)|=1$ and $\|f\|=1$ that $f$ achieves equality in (4.29) for $p=2,3, \ldots$.

However, our $f$ is allowed to have zeros. What the argument of the proof in Ref. 44 shows, though, is that if a smooth $g$ achieves equality in (4.29) and $g\left(q_{1}\right) \neq 0$, then $g$ has the special form (4.30) in a neighborhood of $q_{1}$. And as our $f$ is an entire function, the conclusion that $f$ has the special form (4.30) remains equally valid.

If we calculate $\mathrm{Amb}_{\mathrm{g}}$ for the $g$ of (4.30), we find

$$
\begin{aligned}
\operatorname{Amb}_{g}(\theta, \tau) & \\
= & (1 / 2 \operatorname{Re} \alpha)^{1 / 2} \exp \left(-2 \pi\left[\operatorname{Re} \gamma-(\operatorname{Re} \beta)^{2} / \operatorname{Re} \alpha\right]\right) \\
& \times \exp \left[-\frac{1}{2} \pi \tau^{2} \operatorname{Re} \alpha-\frac{1}{2} \pi(\omega-\tau \operatorname{Im} \alpha)^{2} / \operatorname{Re} \alpha\right. \\
& -(2 \pi i / \operatorname{Re} \alpha)(\omega \operatorname{Re} \beta+\tau \operatorname{Im} \beta \bar{\alpha})] .
\end{aligned}
$$

It is now easy to check from (4.27) that $|\Phi(\theta, \tau)|=1$ implies that $\alpha=1, \beta \in \mathbb{C}$ arbitrary, $\gamma \in \mathbb{C}$ such that $\operatorname{Amb}_{f}(0,0)=1$. Then $\Phi$ becomes

$$
\Phi(\theta, \tau)=\exp [-2 \pi i(\tau \operatorname{Im} \beta+\theta \operatorname{Re} \beta)] \quad\left[(\theta, \tau) \in \mathbb{R}^{2}\right],
$$

and

$$
\varphi(q, p)=\delta(q+\operatorname{Re} \beta) \delta(p+\operatorname{Im} \beta) \quad\left[(q, p) \in \mathbb{R}^{2}\right] .
$$

This completes the proof.

We shall now prove the claim made in connection with (4.16) and (4.17). It is likely that the results of the lemma below for $\gamma \geqslant 1$ are known, but we could not find appropriate references. In addition, we get useful information for the case that $0<\gamma<1$. 
Lemma 4.2: Let $\gamma>0$, and assume that $f \in L^{2}(\mathbb{R})$ satisfies

$$
\begin{aligned}
& \left.f(q)=O\left[\exp \left(-\pi \gamma q^{2}\right)\right] \quad \text { (a.e. } q \in \mathbb{R}\right), \\
& (\mathscr{F} f)(p)=O\left[\exp \left(-\pi \gamma p^{2}\right)\right] \quad(\text { a.e. } p \in \mathbb{R}) .
\end{aligned}
$$

Then, (a) if $\gamma>1$, we have $f=0$, (b) if $\gamma=1$, we have $f(q)=c \exp \left(-\pi q^{2}\right)$ for some $c \in \mathbb{C}$, (c) if $0<\gamma<1$, we have, with $r=(1+\gamma)^{1 / 2}(1-\gamma)^{-1 / 2}$,

$$
\sum_{n=0}^{N}\left|\left(f, \psi_{n}\right)\right|^{2} r^{n}=O(N) \quad(N=0,1, \ldots)
$$

Proof: We obtain from Mehler's formula (2.8), with $-i w$ instead of $w$,

$$
\begin{gathered}
\left(\frac{2}{1+w^{2}}\right)^{1 / 2} \exp \left(-\pi\left(q^{2}+p^{2}\right) \frac{1-w^{2}}{1+w^{2}}-\frac{4 \pi i q p w}{1+w^{2}}\right) \\
=\sum_{n=0}^{\infty}(-i w)^{n} \psi_{n}(q) \psi_{n}(p) \quad\left[(q, p) \in \mathbb{R}^{2},|w|<1\right] .
\end{gathered}
$$

Noting that $\mathscr{F} \psi_{n}=(-i)^{n} \psi_{n}$, multiplying (4.36) by $f(q) \overline{(\mathscr{F} f)(p)}$ and integrating the result over the phase plane, we obtain for $|w|<1$

$$
\begin{aligned}
\sum_{n=0}^{\infty} w^{n}\left|\left(f, \psi_{n}\right)\right|^{2} & \\
= & \left(\frac{2}{1+w^{2}}\right)^{1 / 2} \iint f(q) \overline{\mathscr{F} f)(p)} \\
& \times \exp \left(-\pi\left(q^{2}+p^{2}\right) \frac{1-w^{2}}{1+w^{2}}-\frac{4 \pi i q p w}{1+w^{2}}\right) d q d p
\end{aligned}
$$

We let $w>0$, we insert the estimates (4.34) in the integral at the right-hand side of $(4.37)$, and we take the modulus. The integral that turns up can be evaluated explicitly, and we obtain

$$
\sum_{n=0}^{\infty} w^{n}\left|\left(f, \psi_{n}\right)\right|^{2} \leqslant K \frac{\left(1+w^{2}\right)^{1 / 2}}{\gamma+1+(\gamma-1) w^{2}} \quad(0<w<1),
$$

for some constant $K \geqslant 0$. The integral in (4.37) thus converges absolutely as long as $\gamma+1+(\gamma-1) w^{2}>0$.

Since the left-hand side of (4.37) is a power series with non-negative coefficients, we see by Pringsheim's theorem ${ }^{45}$ that the radius of convergence of the power series is at least equal to $r$ when $0<\gamma<1$, and $\infty$ when $\gamma \geqslant 1$. In the first case we have in addition that

$$
\limsup _{w \rightarrow r-}(r-w) \sum_{n=0}^{\infty} w^{n}\left|\left(f, \psi_{n}\right)\right|^{2}<\infty .
$$

It is not hard to see then that

$$
\sum_{n=0}^{N}\left|\left(f, \psi_{n}\right)\right|^{2} r^{n}=O(N) \quad(N=0,1, \ldots) .
$$

In the case $\gamma>1$ we see that the right-hand side of (4.38) tends to zero when $w \rightarrow \infty$. This implies that $\left(f, \psi_{n}\right)=0$ for all $n$, whence $f=0$. Finally, if $\gamma=1$, we see that the righthand side of $(4.38)$ is $O(|w|), w \rightarrow \infty$, whence $\left(f, \psi_{n}\right) \neq 0$ is only possible for $n=0,1$. Since $\psi_{0}(q)=2^{1 / 4} \exp \left(-\pi q^{2}\right)$, $\psi_{1}(q)=2 \pi^{1 / 2} q \psi_{0}(q)$ we see from $(4.34)$ that $\left(f, \psi_{1}\right)=0$. This completes the proof.
In the remainder of this paper we let $0<\gamma<1$. We shall find conditions on the Wigner distributions of the $f_{n}$ 's as in (4.2) and on $G$ that must be satisfied in order that $(3.1)$ is nonnegative for any $f$ while $C_{f}^{(\Phi)}$ has the correct marginals for any $f$. There exist $\Phi \neq 1$ with these two properties, viz. $\Phi(\theta, \tau)=\exp (\pi \delta \theta \tau) \quad\left[(\theta, \tau) \in \mathbb{R}^{2}\right]$ with $\delta= \pm \gamma^{-1}\left(1-\gamma^{2}\right)^{1 / 2}$. (In fact, this example is not quite proper since $\Phi$ cannot be tested against all elements of $S^{2}$.) It can be shown that the $G$ of (3.3) equals in this case

$$
\begin{aligned}
G(q, p) & =W_{f}(q, p) \\
& =\frac{1}{\gamma} \exp \left(-\frac{2 \pi \gamma\left(q^{2}+p^{2}\right)}{1+\sqrt{1-\gamma^{2}}}-\frac{2 \pi}{\gamma} \sqrt{1-\gamma^{2}}(q+p)^{2}\right) \\
{\left[(q, p) \in \mathbb{R}^{2}\right], } &
\end{aligned}
$$

where

$f(q)=(1 / 2 \gamma)^{1 / 4} \exp \left(-\pi\left[\gamma+i\left(1-\gamma^{2}\right)^{1 / 2}\right] q^{2}\right) \quad(q \in \mathbb{R})$.

Since the collection of all $\Phi$ 's with (3.1) non-negative and (1.7) valid for all $f$ is closed under taking convex combinations, it does not seem easy to describe this collection.

The $f$ in (4.42) satisfies

$$
\begin{aligned}
& |f(q)|=(1 / 2 \gamma)^{1 / 4} \exp \left(-\pi \gamma q^{2}\right) \quad(q \in \mathbb{R}), \\
& |(\mathscr{F} f)(p)|=(1 / 2 \gamma)^{1 / 4} \exp \left(-\pi \gamma p^{2}\right) \quad(p \in \mathbb{R}),
\end{aligned}
$$

while its Wigner distribution satisfies

$$
W_{f}(q, p)=O\left[\exp \left(-\frac{2 \pi \gamma\left(q^{2}+p^{2}\right)}{1+\sqrt{1-\gamma^{2}}}\right)\right] \quad\left[(q, p) \in \mathbb{R}^{2}\right],
$$

and its Hermite coefficients are given by $\left(w=\gamma+i \sqrt{1-\gamma^{2}}\right)$

$$
\left(f, \psi_{k}\right)=0 \text { or } \frac{\sqrt{2 n !}}{(2 \gamma)^{1 / 4} 2^{n} n !}\left(\frac{w-1}{w+1}\right)^{n},
$$

according as $k$ is odd or $k=2 n$ is even. Hence

$$
\left(f, \psi_{k}\right)=O\left(\left|\frac{w-1}{w+1}\right|^{k / 2}\right)=O\left(\left(\frac{1-\gamma}{1+\gamma}\right)^{k / 4}\right) .
$$

See also Theorem 4.3 below.

To find a condition on the $W_{f_{n}}$ 's and on $G$, we recall from the proof of Theorem 4.1 that

$\left(K(q, p)=\exp \left[-2 \pi \gamma\left(q^{2}+p^{2}\right)\right]\right)$

$$
G=\varphi * K=\sum_{n} c_{n} W_{f_{n}},
$$

with $f_{n}$ orthonormal, $c_{n} \geqslant 0, \Sigma_{n} c_{n}<\infty$ and, for $(q, p) \in \mathbb{R}^{2}$,

$$
\begin{aligned}
& \sum_{n} c_{n}\left|f_{n}(q)\right|^{2}=\left(\frac{1}{2 \gamma}\right)^{1 / 2} \exp \left(-2 \pi \gamma q^{2}\right), \\
& \sum_{n} c_{n}\left|\left(\mathscr{F} f_{n}\right)(p)\right|^{2}=\left(\frac{1}{2 \gamma}\right)^{1 / 2} \exp \left(-2 \pi \gamma p^{2}\right) .
\end{aligned}
$$

We shall show that for any $n=0,1, \ldots$ and for any

$$
\begin{gathered}
\epsilon<\gamma /\left(1+\sqrt{\left.1-\gamma^{2}\right)},\right. \\
W_{f_{n}}(q, p)=O\left(\exp \left[-2 \pi \epsilon\left(q^{2}+p^{2}\right)\right]\right) \\
{\left[(q, p) \in \mathbb{R}^{2}\right] .}
\end{gathered}
$$

To that end we prove the following theorem. 
Theorem 4.3: Let $f \in L^{2}(\mathbb{R})$ and consider the following statements: (a) for all $\delta<\gamma$ we have

$$
\begin{aligned}
& f(q)=O\left(e^{-\pi \delta q^{2}}\right) \quad(q \in \mathbb{R}), \\
& (\mathscr{F} f)(p)=O\left(e^{\left.-\pi \delta p^{2}\right)} \quad(p \in \mathbb{R}) ;\right.
\end{aligned}
$$

(b) for all $\delta<\gamma$ we have

$$
\left(f, \psi_{n}\right)=O\left[\left(\frac{1-\delta}{1+\delta}\right)^{n / 4}\right] \quad(n=0,1, \ldots)
$$

and (c) for all $\epsilon<\gamma /\left(1+\sqrt{1-\gamma^{2}}\right)$ we have

$$
W_{f}(q, p)=O\left(\exp \left[-2 \pi \epsilon\left(q^{2}+p^{2}\right)\right]\right) \quad\left[(q, p) \in \mathbb{R}^{2}\right] .
$$

Then $(\mathrm{a}) \Leftrightarrow(\mathrm{b}),(\mathrm{b}) \Leftrightarrow(\mathrm{c})$.

Proof: The implication $(a) \Rightarrow$ (b) follows from Lemma 4.2 (c); in fact the result proved there is slightly more precise. We shall now show that $(b) \Rightarrow(c)$. To that end we assume that $(b)$ holds and we let $0<\delta<\gamma$. We can write $f=N_{\alpha} g$, where $\alpha=\frac{1}{4} \log (1+\delta)(1-\delta)^{-1}$ and where the Hermite coefficients of $g$ equal

$$
\left(g, \psi_{n}\right)=\left(\frac{1+\delta}{1-\delta}\right)^{n / 4+1 / 8}\left(f, \psi_{n}\right) \quad(n=0,1, \ldots) .
$$

Hence $g \in S$. Now, by (2.9) and (2.10),

$$
\begin{aligned}
W_{f}(q, p) & =W_{N_{a g}}(q, p) \\
& =\sqrt{2}\left(N_{\alpha, 2} V_{g}\right)(q \sqrt{2}, p \sqrt{2}) \quad\left[(q, p) \in \mathbb{R}^{2}\right] .
\end{aligned}
$$

The kernel $K_{\alpha, 2}$ of the smoothing operator $N_{\alpha, 2}$ can be written as

$$
\begin{aligned}
& K_{\alpha, 2}(q, p ; x, y)= \frac{1}{\sinh \alpha} \exp \left[-\pi\left(q^{2}+p^{2}\right) \tanh \alpha\right] \\
& \times \exp \left(-\pi\left[(q-x / \cosh \alpha)^{2}\right.\right. \\
&\left.\left.+(p-y / \cosh \alpha)^{2}\right] \operatorname{coth} \alpha\right) \\
& {\left[(q, p ; x, y) \in \mathbb{R}^{2} \times \mathbb{R}^{2}\right] . }
\end{aligned}
$$

Since $V_{g} \in S^{2}$ we easily obtain that

$$
W_{f}(q, p)=O\left(\exp \left[-2 \pi\left(q^{2}+p^{2}\right) \tanh \alpha\right]\right)\left[(q, p) \in \mathbb{R}^{2}\right] .
$$

And as

$$
\tanh \alpha=\frac{e^{2 \alpha}-1}{e^{2 \alpha}+1}=\frac{\delta}{1+\sqrt{1-\delta^{2}}},
$$

the proof of $(b) \Rightarrow(c)$ is complete.

We next show the converse $(c) \Rightarrow(b)$, and therefore we assume that (c) holds. It follows that for $0<\epsilon$ $<\gamma /\left(1+\sqrt{1+\gamma^{2}}\right)$, the integral

$$
\iint \exp \left[2 \pi \epsilon\left(q^{2}+p^{2}\right)\right] W_{f}(q, p) d q d p
$$

converges absolutely. Now let, for $A \geqslant 0$,

$$
\begin{aligned}
& K(r)=e^{\epsilon r} \quad(r \geqslant 0), \\
& K_{A}(r)=\max (K(r), A) \quad(r \geqslant 0) .
\end{aligned}
$$

Then we have by (2.15) (see Ref. 46), for $A \geqslant 0$,

$$
\begin{aligned}
\iint K_{A}\left[2 \pi\left(q^{2}+p^{2}\right)\right] W_{f}(q, p) d q d p \\
=\sum_{n=0}^{\infty}(-1)^{n}\left|\left(f, \psi_{n}\right)\right|^{2} \int_{0}^{\infty} e^{-r} K_{A}(r) L_{n}(2 r) d r .
\end{aligned}
$$

Since $K_{A}$ is nondecreasing we can apply Theorem 2.1, and we find that

$$
\begin{gathered}
c_{n}(A):=(-1)^{n} \int_{0}^{\infty} e^{-r} K_{A}(r) L_{n}(2 r) d r \geqslant 0 \\
(A \geqslant 0, n=0,1, \ldots) .
\end{gathered}
$$

Also, by the generating function of the Laguerre polynomials,

$$
\begin{aligned}
\lim _{A \rightarrow \infty} c_{n}(A) & =(-1)^{n} \int_{0}^{\infty} e^{-r} K(r) L_{n}(2 r) d r \\
& =(1-\epsilon)^{n-1} /(1+\epsilon)^{n} \quad(n=0,1, \ldots) .
\end{aligned}
$$

Since the left-hand side of $(4.60)$ tends to the finite number in (4.58) as $A \rightarrow \infty$, we easily conclude that

$$
\sum_{n=0}^{\infty} \frac{(1-\epsilon)^{n-1}}{(1+\epsilon)^{n}}\left|\left(f, \psi_{n}\right)\right|^{2}<\infty .
$$

The proof is completed by noting that $(1-\epsilon)^{1 / 2}$ $\times(1+\epsilon)^{-1 / 2}=(1-\delta)^{1 / 4}(1+\delta)^{-1 / 4}$ when $\epsilon=\delta /$ $\left(1+\sqrt{1-\delta^{2}}\right)$.

Note: Assume that $f$ satisfies (c). Then it follows from (1.7) that (a) is satisfied with $\gamma$ replaced by $\gamma /\left(1+\sqrt{1-\gamma^{2}}\right)$. The implication $(\mathrm{a}) \Rightarrow(\mathrm{b})$ cannot be strengthened [see (4.43)(4.45)].

We conclude this paper with the following theorem.

Theorem 4.4: Let $G$ be as in (4.46). Then we have

$$
G(q, p)=O\left(\exp \left[-2 \pi \epsilon\left(q^{2}+p^{2}\right)\right]\right) \quad\left[(q, p) \in \mathbb{R}^{2}\right](4.64)
$$

for all $\epsilon<\gamma /\left(1+\sqrt{1-\gamma^{2}}\right)$.

Proof: The proof follows rather closely the proof of the statements $(a) \Rightarrow(b),(b) \Rightarrow(c)$ in Theorem 4.3 . Therefore we shall omit details.

Let $\widetilde{G}(q, p):=(1 / \sqrt{2}) G(q / \sqrt{2}, p / \sqrt{2})$, and define

$$
\begin{aligned}
& W_{f, g}(q, p) \\
& =\int e^{-2 \pi i p t} f\left(q+\frac{1}{2} t\right) \overline{g\left(q-\frac{1}{2} t\right)} d t \quad\left[(q, p) \in \mathbb{R}^{2}\right],(4) \\
& V_{f, g}(q, p)=\frac{1}{\sqrt{2}} W_{f, g}\left(\frac{q}{\sqrt{2}}, \frac{p}{\sqrt{2}}\right) \quad\left[(q, p) \in \mathbb{R}^{2}\right]
\end{aligned}
$$

for $f \in S, g \in S$. Then we have $\left(G, W_{f, g}\right)=\left(\widetilde{G}, V_{f, g}\right)$ for all $f \in S$, $g \in S$. have

We shall estimate the Hermite coefficients of $\widetilde{G}$. We

$$
\left(\widetilde{G}, \psi_{k} \otimes \psi_{l}\right)=\sum_{i, j}\left(\widetilde{G}, V_{\psi_{i} \psi_{j}}\right) \gamma_{i j, k l},
$$

with

$$
\gamma_{i j, k l}=\left(V_{\psi_{i}, \psi_{j}}, \psi_{k} \otimes \psi_{l}\right)
$$

This follows from completeness and orthonormality of $\left(V_{\psi_{i} \psi_{j}}\right)_{i, j}$ in $L^{2}\left(\mathbf{R}^{2}\right)$ (see also the proof of Lemma 4.1). According to Ref. 8, 27.26.1, $\gamma_{i j, k l}$ equals $a_{i}^{-1} a_{j}^{-1} a_{k} a_{l}$ times the coefficient of $w^{i} z^{j}$ in $[(w+z) / \sqrt{2}]^{k}[(w-z) / i \sqrt{2}]^{l}$; here $a_{n}=(n !)^{-1 / 2} 2^{-1 / 4}(4 \pi)^{n / 2}$. It is important to observe that $\gamma_{i j, k l}=0$, when $k+l \neq i+j$.

It is easy to see that, for all $i, j$, 


$$
\left|\left(\widetilde{G}, V_{\psi_{i} \psi_{j}}\right)\right|^{2}=\left|\left(G, W_{\psi_{i} \psi_{j}}\right)\right|^{2} \leqslant\left(G, W_{\psi_{i}}\right)\left(G, W_{\psi_{j}}\right),
$$

whence, as $\Sigma_{i, j}\left|\gamma_{i j, k l}\right|^{2}=\left\|\psi_{k} \otimes \psi_{l}\right\|^{2}=1$,

$$
\left|\left(\widetilde{G}, \psi_{k} \otimes \psi_{l}\right)\right|^{2} \leqslant \sum_{i+j=k+l}\left(G, W_{\psi_{i}}\right)\left(G, W_{\psi_{j}}\right),
$$

by the Cauchy-Schwarz inequality.

To estimate $\left(G, W_{\psi_{i}}\right)$, we consider $\Sigma_{k=0}^{\infty} w^{k}\left(G, W_{\psi_{k}}\right)$ $=: F(w)$ for $|w|<1$. We have, as in the proof of Lemma 4.1, for $|w|<1$,

$$
\begin{aligned}
F(w)= & \left(\frac{2}{1+w^{2}}\right)^{1 / 2} \iint H(q, p) \\
& \times \exp \left(-\pi\left(q^{2}+p^{2}\right) \frac{1-w^{2}}{1+w^{2}}-\frac{4 \pi i q p w}{1+w^{2}}\right) d q d p,
\end{aligned}
$$

with

$$
H(q, p)=\sum_{n} c_{n} f_{n}(q) \overline{\left(\mathscr{F} f_{n}\right)(p)} \quad\left[(q, p) \in \mathbb{R}^{2}\right] .
$$

It follows easily from the Cauchy-Schwarz inequality and (4.43) and (4.44) that

$$
|H(q, p)| \leqslant\left(\frac{1}{2 \gamma}\right)^{1 / 2} \exp \left[-\pi \gamma\left(q^{2}+p^{2}\right)\right] \quad\left[(q, p) \in \mathbb{R}^{2}\right] .
$$

As in the proof of Lemma 4.1(c) we conclude that

$$
\sum_{k=0}^{N} r^{k}\left(G, W_{\psi_{k}}\right)=O(N) \quad(N=0,1, \ldots),
$$

where $r=(1+\gamma)^{1 / 2}(1-\gamma)^{-1 / 2}$. Hence $\left(G, W_{\psi_{k}}\right)$ $=O\left([(1-\delta) /(1+\delta)]^{k / 2}\right)$ for all $\delta<\gamma$, and we obtain by (4.70), for all $\delta<\gamma$,

$$
\left(\widetilde{G}, \psi_{k} \otimes \psi_{l}\right)=O\left[\left(\frac{1-\delta}{1+\delta}\right)^{(k+l) / 4}\right] \quad(k, l=0,1, \ldots) .
$$

This shows that for any $\alpha<\frac{1}{4} \log [(1+\gamma) /(1-\gamma)]$ there is an $F \in S^{2}$ such that $\widetilde{G}=N_{\alpha, 2} F$. As in the proof of the statement $(b) \Rightarrow(c)$ in Theorem 4.3 we conclude that, for any $\alpha<\frac{1}{4} \log [(1+\gamma) /(1-\gamma)]$,

$$
\widetilde{G}(q, p)=O\left(\exp \left[-\pi\left(q^{2}+p^{2}\right) \tanh \alpha\right]\right) \quad\left[(q, p) \in \mathbb{R}^{2}\right],
$$

and the proof is easily completed now.

\section{ACKNOWLEDGMENTS}

The author thanks T. A. C. M. Claasen and W. F. G. Mecklenbräuker, who anticipated some of the results of this paper, for asking the right questions.

\section{APPENDIX A: SMOOTH POSITIVE DEFINITE FUNCTIONS OF TWO VARIABLES}

In the proof of Theorem 4.2 the following theorem was required.

Theorem A.1: Let $K \in S^{2}$ be positive definite, i.e., $(K, f \otimes \bar{f}) \geqslant 0$ for all $f \in L^{2}(\mathbb{R})$. There are non-negative numbers $c_{n}$ and orthonormal $f_{n} \in S$ such that

$$
K(q, p)=\sum_{n} c_{n} f_{n}(q) \overline{f_{n}(p)} \quad\left[(q, p) \in \mathbb{R}^{2}\right],
$$

with convergence in the $S^{2}$ sense. Moreover, when the $c_{n}$ 's are ordered decreasingly we have $c_{n}=O\left(e^{-n \epsilon}\right)$ for some $\epsilon>0$. lemma.

The proof of this theorem relies on the following

Lemma A.1: Let $K_{n} \in S^{2}(n=0,1, \ldots)$. Then $K_{n} \rightarrow 0$ in the $S^{2}$ sense if and only if $\left(K_{n}, F \otimes \bar{F}\right) \rightarrow 0$ for every $F \in S^{*}$.

Proof: It is known ${ }^{47}$ that $K_{n} \rightarrow 0$ in the $S^{2}$ sense if and only if $\left(K_{n}, H\right) \rightarrow 0$ for all $H \in S^{2 *}$. Hence we only have to show that $\left(K_{n}, F \otimes \bar{F}\right) \rightarrow 0$ for every $F \in S^{*}$ implies that $\left(K_{n}, H\right) \rightarrow 0$ for every $H \in S^{2 *}$.

By polarization we can assume that $\left(K_{n}, F \otimes G\right) \rightarrow 0$ for every $F \in S^{*}, G \in S^{*}$. Let $F \in S^{*}$. The space $S^{*}$ is a Fréchet space $^{48}$; as a countable system of norms on $S^{*}$ we can take, for $m=1,2, \ldots$,

$$
\|G\|_{m}=\left(\sum_{k=0}^{\infty}\left|\left(G, \psi_{k}\right)\right|^{2} e^{-2 k / m}\right)^{1 / 2}\left(G \in S^{*}\right) .
$$

Therefore we can find, by boundedness of

$\left(K_{n}, F \otimes G\right)(n=0,1, \ldots)$ for every $G \in S^{*}$, an $m=1,2, \ldots$ and an $M>0$ such that

$$
\left|\left(K_{n}, F \otimes G\right)\right| \leqslant M \quad(n=0,1, \ldots)
$$

for all $G \in S^{*}$ with $\|G\|_{m} \leqslant 1$. Hence, $S^{*}=\cup_{l=1}^{\infty} B_{l}$, where $B_{l}=\left\{F \in S^{*}\left|\|G\|_{l} \leqslant 1 \Rightarrow\right|\left(K_{n}, F \otimes G\right) \mid \leqslant l(n=0,1, \ldots)\right\}$,

for $l=1,2, \ldots$. Again using that $S^{*}$ is a Fréchet space we conclude that there is an $l_{0}=1,2, \ldots$ and an open set in $S^{*}$ in which $B_{l_{0}}$ is dense. From this we infer the existence of $M>0$, $k_{0}=1,2, \ldots$ with

$$
\left|\left(K_{n}, F \otimes G\right)\right| \leqslant M \quad(n=0,1, \ldots),
$$

for all $F \in S^{*}, G \in S^{*}$ with $\|F\|_{k_{0}} \leqslant 1,\|G\|_{\iota_{0}} \leqslant 1$. If we take $F=\exp \left(k / k_{0}\right) \psi_{k}, G=\exp \left(l / l_{0}\right) \psi_{1}$, we get

$\left|\left(K_{n}, \psi_{k} \otimes \psi_{l}\right)\right| \leqslant M \exp \left(-k / k_{0}-l / l_{0}\right) \quad(n, k, l=0,1, \ldots)$.

It is now easy to show [as $\left(K_{n}, \psi_{k} \otimes \psi_{1}\right) \rightarrow 0$ for all $\left.k, l\right]$ that $\left(K_{n}, H\right)=\Sigma_{k, l}\left(K_{n}, \psi_{k} \otimes \psi_{l}\right)\left(\psi_{k} \otimes \psi_{l}, H\right) \rightarrow 0$ for every $H \in S^{2 *}$.

Corollary: With an entirely similar proof one can show that if $K_{n} \in S^{2}$ and $\lim \left(K_{n}, F \otimes \bar{F}\right)$ exists for all $F \in S^{*}$ then there is exactly one $K \in S^{2}$ with $K_{n} \rightarrow K$ in the $S^{2}$ sense. $\operatorname{tion}^{49}$

We now prove Theorem A.1. We have the representa-

$$
K=\sum_{n} c_{n} f_{n} \otimes \bar{f}_{n},
$$

where $c_{n} \geqslant 0, \Sigma_{n} c_{n}^{2}<\infty, f_{n} \in L^{2}(\mathbb{R})$ orthonormal and where the convergence is in the $L^{2}\left(\mathbb{R}^{2}\right)$ sense. In addition, for every $n$,

$$
c_{n} f_{n}(u)=\int K(u, v) f_{n}(v) d v \quad(u \in \mathbb{R}),
$$

and from this one readily concludes that $f_{n} \in S$, e.g., by expanding $K$ in a Hermite series $\Sigma_{k, l} d_{k l} \psi_{k} \otimes \psi_{l}$ with $d_{k l}=O(\exp [-\epsilon(k+l)])$ for some $\epsilon>0$. We assume here and in the remainder that $c_{n}>0$.

Now let $F \in S^{*}$. We shall check that $\Sigma_{n} c_{n}\left|\left(f_{n}, F\right)\right|^{2}<\infty$. To that end we take a sequence $F_{k}$ in $S$ with $F_{k} \rightarrow F$ in the $S^{*}$ sense if $k \rightarrow \infty$. We have, for all $k$,

$$
\left(K, F_{k} \otimes \bar{F}_{k}\right)=\sum_{n} c_{n}\left|\left(f_{n}, F_{k}\right)\right|^{2},
$$


by (A6). The terms in the right-hand side series are nonnegative for all $k$ and tend to $c_{n}\left|\left(f_{n}, F\right)\right|^{2}$ when $k \rightarrow \infty$. The left-hand side tends to $(K, F \otimes \bar{F})$, when $k \rightarrow \infty$. By Fatou's lemma we conclude that $\Sigma_{n} c_{n}\left|\left(f_{n}, F\right)\right|^{2}<\infty$. That is, we have shown that $\lim _{N \rightarrow \infty}\left(\sum_{n=0}^{N} c_{n} f_{n} \otimes \bar{f}_{n}, F \otimes \bar{F}\right)$ exists for all $F \in S^{*}$. The corollary after Lemma A.1 implies that $\Sigma_{n=0}^{N} c_{n} f_{n} \otimes \bar{f}_{n}$ converges in the $S^{2}$ sense. Because of (A7) the limit is $K$, whence $K=\Sigma_{n} c_{n} f_{n} \otimes \bar{f}_{n}$ with convergence in the $S^{2}$ sense.

We finally show that $c_{n}=0\left(e^{-n \epsilon}\right)$ for some $\epsilon>0$. It is assumed here that $c_{n} \geqslant c_{n+1}>0($ all $n)$. We have

$$
\left(K, \psi_{k} \otimes \psi_{k}\right)=\sum_{n} c_{n}\left|\left(f_{n}, \psi_{k}\right)\right|^{2}=O\left(e^{-2 k \epsilon}\right)
$$

for some $\epsilon>0$. Hence there is an $M>0$ such that, for all $n$,

$$
c_{n} \sum_{k}\left|\left(f_{n}, \psi_{k}\right)\right|^{2} e^{k \epsilon} \leqslant M .
$$

It follows from orthonormality of the $f_{n}$ 's and Parseval's theorem that for any $m=1,2, \ldots$ there is an $n=n(m)=0,1, \ldots, m+1$ such that

$$
\sum_{k=m+1}^{\infty}\left|\left(f_{n}, \psi_{k}\right)\right|^{2} \geqslant \frac{1}{m+2} \text {. }
$$

Therefore, $c_{n}(m) \leqslant M(m+2) e^{-(m+1) \epsilon}$.

We have assumed that $c_{n}>0$ for all $n$, and therefore $n(m) \rightarrow \infty$ as $m \rightarrow \infty$. Now let $n=1,2, \ldots$, and take an $m$ with $n(m) \leqslant n \leqslant n(m+1)$. Then $m \geqslant n-2$, and, by monotonicity of the $c_{n}$ 's,

$$
c_{n} \leqslant c_{n(m)} \leqslant M(m+2) e^{-(m+1) \epsilon} \leqslant M n e^{-(n-1) \epsilon},
$$

when $n$ is sufficiently large. This completes the proof of Theorem A.1.

\section{APPENDIX B: SECOND PROOF OF THEOREM 4.2 (b)}

We start from the formula $\varphi * K=\frac{1}{2} W_{f}$ in (4.26), where $\varphi$ satisfies $\varphi * \tilde{\varphi}=\delta \otimes \delta, K(q, p)=\exp \left[-2 \pi\left(q^{2}+p^{2}\right)\right]$, and $f \in S,\|f\|=1$. This formula can also be written as $\varphi * W_{g}=W_{f}$, where $g(q)=2^{1 / 4} \exp \left(-\pi q^{2}\right)$.

We shall use the following result ${ }^{50}$ : when $\varphi_{0}$ and $\psi_{0}$ are entire functions, then $(z=x+i y)$

$$
\begin{gathered}
2 \iint_{\mathbb{C}}\left|\varphi_{0}(z) \psi_{0}(z)\right|^{2} \exp \left(-2 \pi|z|^{2}\right) d x d y \\
\leqslant \iint_{\mathbb{C}}\left|\varphi_{0}(z)\right|^{2} \exp \left(-\pi|z|^{2}\right) d x d y \\
\quad \times \iint_{\mathbb{C}}\left|\psi_{0}(z)\right|^{2} \exp \left(-\pi|z|^{2}\right) d x d y,
\end{gathered}
$$

and, if the right-hand side is finite, there is equality in (B1) if and only if $\varphi_{0}(z) \psi_{0}(z)$ can be expressed as $C \exp (2 \pi \bar{u} z)$ for some $u \in \mathbb{C}$ and some $C \in \mathbb{C}$. We apply this result with $\varphi_{0}=\psi_{0}=B f$ where $B f$ is the Bargmann transform ${ }^{51}$ of $f$, given by

$$
\begin{aligned}
(B f)(z) & =e^{(1 / 2) \pi z^{2}}(f * g)(z) \\
& =2^{1 / 4} \int e^{(1 / 2) \pi z^{2}-\pi(z-q)^{2}} f(q) d q(z \in \mathbb{C}) .
\end{aligned}
$$

The Bargmann transform provides an isometry between the spaces $L^{2}(\mathbb{R}, d q)$ and $\left.L^{2}\left[\mathbb{C}, \exp \left(-\pi|z|^{2}\right) d x d y\right)\right]$. Hence, the right-hand side of (B1) equals 1 , as $\|f\|=1$. We shall show that the left-hand side of (B1) equals 1 as well, so that $[(B f)(z)]^{2}$ has the special form as indicated above.

According to Ref. 23, Eq. (2.8), we have $(z=x+i y)$

$$
(B f)(z) \exp \left(-\frac{1}{2} \pi|z|^{2}\right)=\left(f, G_{1}(x,-y)\right) \text {, }
$$

where, for $(a, b) \in \mathbb{R}^{2}$,

$$
\begin{aligned}
& G_{1}(a, b)(q) \\
& \quad=2^{1 / 4} \exp \left[-\pi(q-a)^{2}+2 \pi i b q-\pi i a b\right] \quad(q \in \mathbb{R}) .
\end{aligned}
$$

Hence, the left-hand side of $(\mathrm{B} 1)$ can be brought into the form

$$
2 \iint\left|\left(f, G_{1}(x, y)\right)\right|^{4} d x d y
$$

By Moyal's formula we have

$$
\begin{aligned}
& \left|\left(f, G_{1}(x, y)\right)\right|^{2} \\
& =2 \iint W_{f}(a, b) \exp \left[-2 \pi(x-a)^{2}\right. \\
& \left.\quad-2 \pi(y-b)^{2}\right] d a d b=\left(W_{f} * W_{g}\right)(x, y) .
\end{aligned}
$$

Hence, the left-hand side of $(\mathrm{B} 1)$ can be written as

$$
2\left(W_{f} * W_{g}, W_{f} * W_{g}\right)
$$

Now $W_{f}=\varphi * W_{g}$, and $\left(\varphi * H_{1}, \varphi * H_{2}\right)$ $=\left(\varphi * \varphi * H_{1}, H_{2}\right)=\left(H_{1}, H_{2}\right)$ for any $H_{1} \in S^{2}, H_{2} \in S^{2}$. Hence, the left-hand side of (B1) equals $2\left(W_{g} * W_{g}, W_{g} * W_{g}\right)$. Using that

$$
\left(W_{g} * W_{g}\right)(a, b)=\exp \left[\left(-\pi\left(a^{2}+b^{2}\right)\right] \quad\left[(a, b) \in \mathbb{R}^{2}\right],\right.
$$

we see that the left-hand side of (B1) equals 1 .

This shows that there is equality in $(\mathbf{B} 1)$, whence $(B f)(z)$ is of the form $C \exp (2 \pi \bar{u} z)$ for some $C \in \mathbb{C}$ and some $u \in \mathbb{C}$. Writing $\bar{u}=a+i b$, we see from Ref. 23 , Eq. (2.8), that $f$ is a multiple of $G_{1}(a, b)$. And since $\|f\|=G_{1}(a, b)=1$, we get

$$
\begin{aligned}
& W_{f}(q, p)=2 \exp \left[-2 \pi(q-a)^{2}-2 \pi(p-b)^{2}\right] \\
& \quad\left[(a, b) \in \mathbb{R}^{2}\right] .
\end{aligned}
$$

Finally the formula $\varphi * W_{f}=W_{g}$ shows that $\varphi(q, p)=\delta(q+a) \delta(p+b)$. This completes the proof.

'L. Cohen, J. Math. Phys. 7, 781 (1966).

${ }^{2}$ E. Wigner, Phys. Rev. 40, 749 (1932).

${ }^{3}$ T. A. C. M. Claasen and W. F. G. Mecklenbräuker, Philips J. Res. 35, 372 (1980).

${ }^{4} \mathrm{H}$. Margenau and L. Cohen, in Probabilities in Quantum Mechanics, Quantum Theory and Reality, edited by M. Bunge (Springer-Verlag, Berlin, 1967), Chap. 4

${ }^{5}$ R. P. Boas, Entire Functions (Academic, New York, 1954).

${ }^{6}$ J. E. Moyal, Proc. Cambridge Philos. Soc. 45, 99 (1949).

${ }^{7}$ H. J. Groenewold, Physica The Hague 12, 405 (1946).

${ }^{8}$ N. G. de Bruijn, Nieuw Arch. Wiskunde 21, 205 (1973).

${ }^{9}$ A. J. E. M. Janssen, Philips J. Res. 37, 79 (1982).

${ }^{10}$ H. Margenau and R. N. Hill, Progr. Theoret. Phys. (Kyoto) 26, 722 (1961).

${ }^{11}$ It is not hard to check that $C_{f}^{(\Phi)}(q, p) \in \mathbb{R}$ for all $(q, p) \in \mathbb{R}^{2}$ and all states $f$ if and only if the $\varphi$ of $(1.6)$ is real.

${ }^{12}$ E. P. Wigner, in Quantum-Mechanical Distribution Functions Revisited, 
Perspectives in Quantum Theory, edited by W. Yourgrau and A. van der Merwe (Dover, New York, 1979), Chap. 4.

${ }^{13}$ L. Cohen and Y. I. Zaparovanny, J. Math. Phys. 21, 794 (1980).

${ }^{14}$ K. Husimi, Proc. Phys. Math. Soc. Jpn. 22, 264 (1940).

${ }^{15}$ N. G. de Bruijn, in Uncertainty Principles in Fourier Analysis, Inequalities, edited by O. Shisha (Academic, New York, 1967).

${ }^{16}$ A. J. E. M. Janssen, SIAM J. Math. Anal. 12, 752 (1981).

${ }^{17}$ R. L. Hudson, Rep. Math. Phys. 6, 249 (1974)

${ }^{18}$ F. Soto and P. Claverie, Physica A 109, 193 (1981).

${ }^{19}$ W. M. de Muynck, P. A. E. M. Janssen, and A. Santman, Found Phys. 9, $71(1979)$.

${ }^{20}$ N. D. Cartwright, Physica A 83, $210(1976)$.

${ }^{21}$ A. J. E. M. Janssen, SIAM J. Math. Anal. 15, 170 (1984).

${ }^{22}$ This condition is satisfied, e.g., when $C_{f}^{(\Phi)}$ has the finite support properties (1.11) and (1.12).

${ }^{23}$ A. J. E. M. Janssen, J. Math. Phys. 23, 720 (1982).

${ }^{24}$ See Ref. 8, Sec. 10.

${ }^{25}$ See Ref. 8, Sec. 25.

${ }^{26}$ See Ref. 8, Sec. 27.12.2.

${ }^{27}$ See Ref. 16, Sec. 2.2; see also Ref. 7 and J. Peetre, Matematiche (Catania) 27, 301 (1972).

${ }^{28}$ See G. Szegö, Orthogonal Polynomials, (AMS, Providence, RI, 1975), 4th ed., Vol. 23.

${ }^{29}$ See Ref. 16, Sec. 2.3 .

${ }^{30}$ T. M. Apostol, Mathematical Analysis (Addison-Wesley, Reading, MA, 1974), 2nd ed., p. 165.

${ }^{31}$ See Ref. 28, 8.4.

${ }^{32}$ See Ref. 8 , Sec. 26

${ }^{33}$ A. J. E. M. Janssen, Proc. K. N. A. W., Ser. A 82, 283 (1979).

${ }^{34}$ See Ref. 28 , Chap. VI, Sec. 8.

${ }^{35}$ See Ref. 23, Eqs. (2.6) and (2.8).
${ }^{36}$ J. C. T. Pool, J. Math. Phys. 7, 66 (1966).

${ }^{37}$ A. C. Zaanen, Linear Analysis (North-Holland, Amsterdam, 1953).

${ }^{38}$ See Ref. 8, Theorem 23.2.

${ }^{39}$ A. J. E. M. Janssen, “Application of the Wigner Distribution to Harmonic Analysis of Generalized Stochastic Processes," MC-tract 114, Mathematisch Centrum, Amsterdam, 1979, Appendix 1, 1.6.

${ }^{40}$ See Ref. 5. By Hartogs' theorem a $\Phi$ satisfying (1.13) and (1.14) is an entire function in both variables, see, e.g., V. S. Vladimirov, in Methods of the Theory of Functions of Many Complex Variables, edited by L. Ehrenpreis (M. I. T., Cambridge, MA, 1966).

${ }^{41}$ This condition means that $\varphi \in \mathscr{C}^{2}$, the class of all elements of $S^{2 *}$ for which $\varphi * f \in S^{2}$ for all $f \in S^{2}$ (see Ref. 33). For such a $\varphi$ the convolution $\varphi * F$ with $F \in S^{2 *}$ makes sense and yields an element of $S^{2 *}$.

${ }^{42}$ The convolution mapping $f \in S^{2} \rightarrow \varphi * f \in S^{2}$ is continuous. See Ref. 33, Theorem 2.3 (i).

${ }^{43}$ P. M. Woodward, Probability and Information Theory, with Applications to Radar (McGraw-Hill, New York, 1953), p. 120

${ }^{44}$ R. Price and E. M. Hofstetter, IEEE Trans. Inf. Theory IT-11, 207 (1965).

${ }^{45} \mathrm{E}$. C. Titchmarsh, The Theory of Functions (Oxford U. P., New York, 1939), 2nd ed.

${ }^{46}$ We cannot use (2.15) directly for $K$ since (2.15) was derived in Ref. 16 for $f \in S$ and measurable $K:[0, \infty) \rightarrow \mathbb{R}$ with $K(r)=O$ [exp $(\epsilon r)]$ for all $\epsilon>0$.

${ }^{47}$ See Ref. 39, Appendix 1, Sec. 3.

${ }^{48}$ See Ref. 39, Appendix 1, Sec. 2.

${ }^{49}$ See Ref. 37.

${ }^{50}$ S. Saitoh, Proc. Am. Math. Soc. 80, 254 (1980), Theorem 1.1, with $\varphi_{0}(z)=\varphi(z \sqrt{\pi}), \psi_{0}(z)=\psi(z \sqrt{\pi})$. The inequality given in this theorem is to some extent equivalent to the inequality in Ref. 44 which was used in the first proof of Theorem 4.2(b) of our paper.

${ }^{51}$ See Ref. 23. 